\title{
Chapter
}

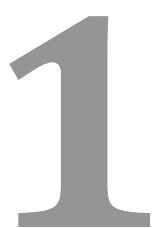

\section{ENZYMES CATALYSING THE SYNTHESIS AND DEGRADATION OF BETA-LINKED BIOPOLYMERS AND THEIR APPLICATIONS}

\section{Tomohiro Hiraishi ${ }^{*}$ and Seiichi Taguchi ${ }^{2}$}

${ }^{1}$ Bioengineering Laboratory, RIKEN, 2-1 Hirosawa, Wako-shi, Saitama 351-0198, Japan

2 Department of Chemistry for Life Sciences and Agriculture, Faculty of Life Sciences, Tokyo University of Agriculture, 1-1-1 Sakuragaoka, Setagaya-ku, Tokyo 155-8502, Japan 


\section{Contents}

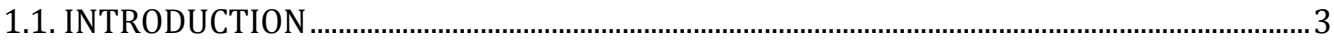

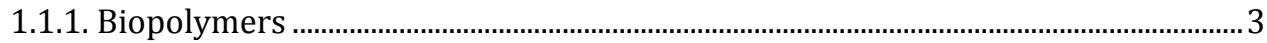

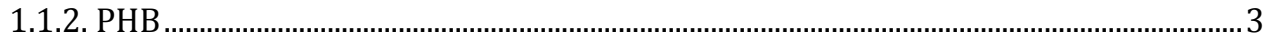

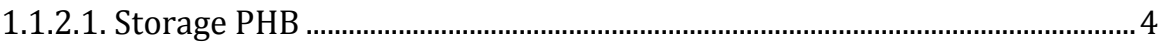

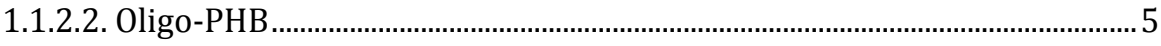

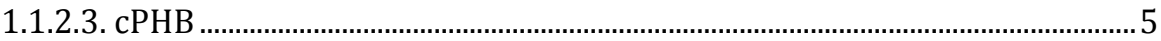

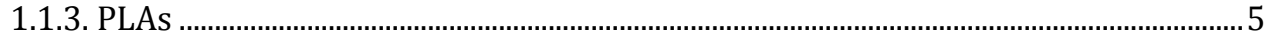

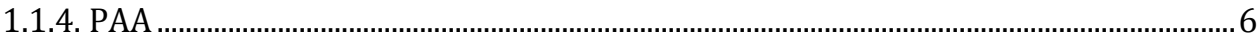

1.1.5. Towards an enhanced sustainable production.............................................................. 6

1.2. PROTEIN ENGINEERING OF PHA SYNTHASES ……............................................................. 7

1.2.1. Enzymology and engineering of PHA synthases ..................................................... 7

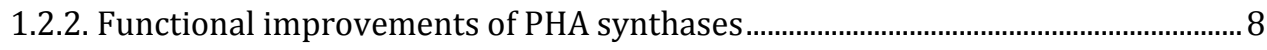

1.2.2.1. Type I PHA synthases ............................................................................... 8

1.2.2.2. Type II PHA synthases.................................................................................. 10

1.2.3. Creation of lactate-polymerizing enzyme (LPE) ...................................................... 11

1.3. PROTEIN ENGINEERING OF PHB DEPOLYMERASE ............................................................ 14

1.3.1. Biochemical and genetic properties of PHB depolymerases ................................... 14

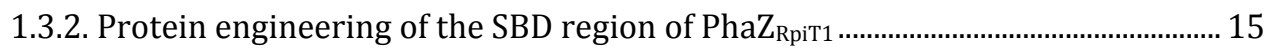

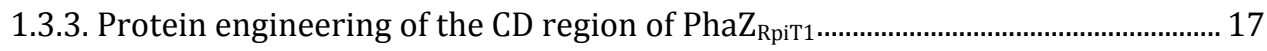

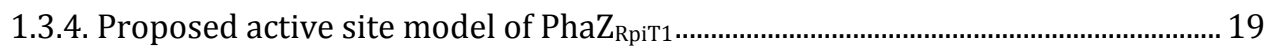

1.3.5. Applications of PHB depolymerases........................................................................ 19

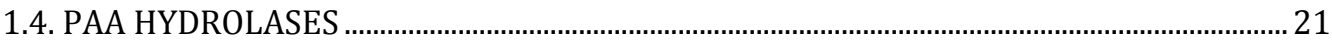

1.4.1. Biochemical and genetic properties of PAA hydrolases .......................................... 21

1.4.2. Enzymes functionally and structurally related to PahZ1 $1_{\mathrm{KT}-1}$ and

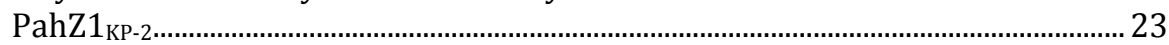

1.4.3. Application of PAA hydrolase from Pedobacter sp. KP-2 ….................................... 26

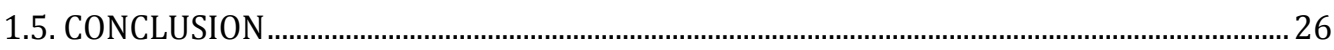

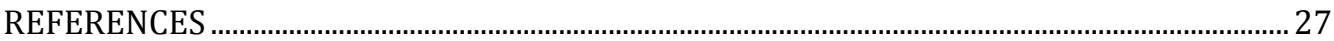




\subsection{INTRODUCTION}

\subsubsection{Biopolymers}

Biopolymers include biodegradable and biobased polymers (Figure 1) [1,2]. The former are produced from renewable or petroleum resources via biological or chemical processes and are degraded by enzymes and microorganisms in natural environments. The latter are synthesized from renewable resources via biological or chemical processes, and some are non-biodegradable, although biobased polymers are generally biodegradable. Poly( $\varepsilon$-caprolactone) (PCL), poly(ethylene succinate) (PES) and poly(butylene succinate) (PBS) are synthesized from petroleum resources via chemical processes, but they have excellent biodegradability. Currently, cost-effective processes for the production of succinic acid and 1,4-butanediol, raw materials of PBS, from biomass resources are being developed. Polyethylene (PE) and polypropylene (PP) are chemically synthesized from their monomers, which can be derived from biological sources, but they are not biodegradable. Poly(hydroxyalkanoate)s (PHAs) and poly(lactide) (PLA) have excellent biodegradability and are produced from renewable resources via biological and chemical processes, respectively. These polymers are water-insoluble and plastic polymers. In contrast, poly(Glu) (PGA) and poly(Asp) (PAA) are watersoluble, biodegradable and biobased polymers. PGA is synthesized via a biological process by several bacteria (all Gram-positive), one archaea and one eukaryote [3], while PAA is synthesized from L-Asp via a chemical process [4]. Accordingly, biodegradable biobased biopolymers, such as PHAs, PLA, PGA and PAA, are the most favourable biopolymers to avoid the environmental problems associated with the use of petrochemical-based synthetic polymers. Among these biobased and biodegradable polymers, this contribution mainly focuses on PHAs and PAA, which contain a novel $\beta$-linkage in the main chain of their molecules, and the enzymes involved in their metabolism in nature.

\subsubsection{PHB}

Poly[(R)-3-hydroxybutyrate] (PHB) is a linear polymer of $R$-3-hydroxybutyrate (3HB) units connected via $\beta$-ester linkage that was first discovered in the mid1920 s by Lemoigne [5]. PHB is categorized into three types in accordance with the number of $3 \mathrm{HB}$ units and biological function: (i) high molecular weight PHB (1,000 to $>1,000,000$ 3HB units, storage PHB), (ii) low molecular weight PHB ( $\approx 100-2003 \mathrm{HB}$ units, oligo-PHB), and (iii) conjugated PHB $(<303 \mathrm{HB}$ units, cPHB) [6-8]. Storage PHB is found in many prokaryotes, such as Eubacteria and Archaea, while oligo-PHB and cPHB are found in both prokaryotes and eukaryotes, that is, oligo-PHB and cPHB may be present in all organisms. 


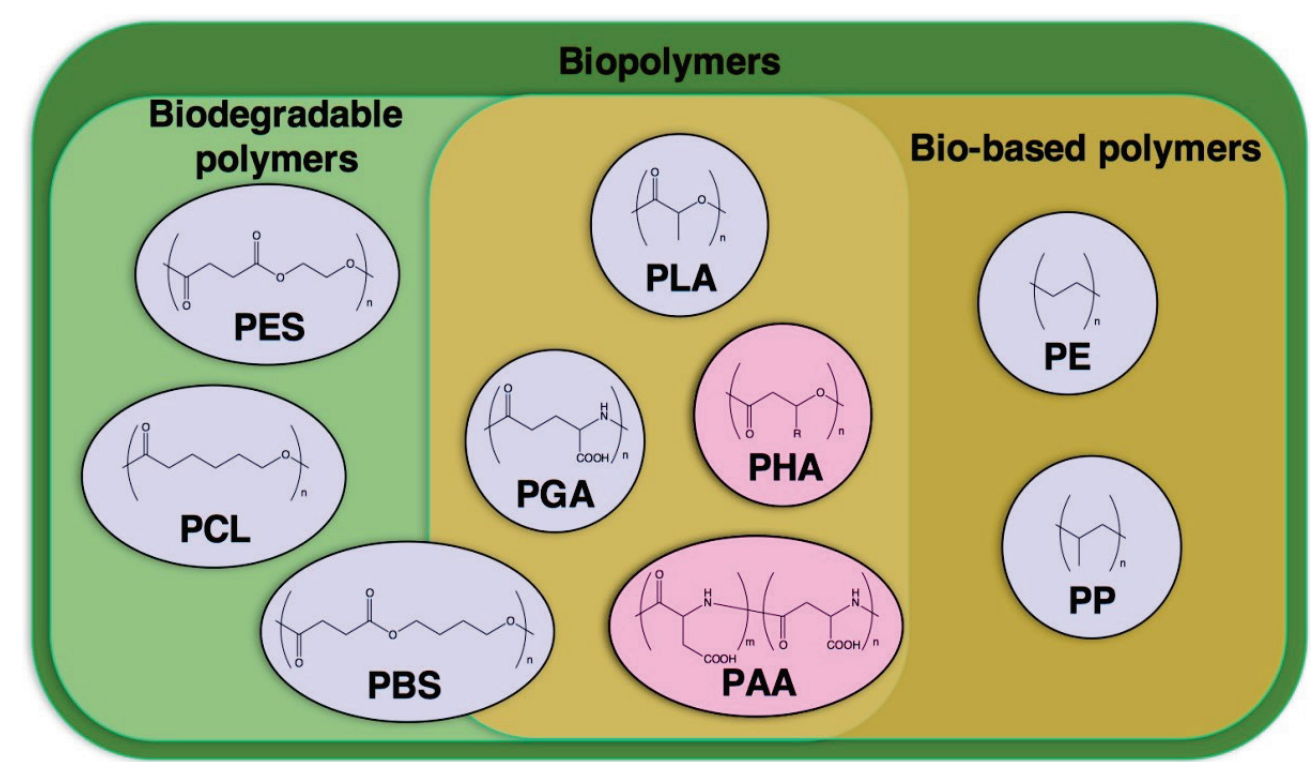

Figure 1. Biopolymers comprised of biodegradable and bio-based polymers

\subsubsection{Storage $P H B$}

Storage PHB and its copolymers (PHAs), including other hydroxyalkanoates (HAs), are intracellularly deposited in the form of inclusion bodies (PHB/PHA granules). In most organisms, PHAs are accumulated as amorphous granules from renewable resources under unbalanced growth conditions, and their surfaces are covered by a layer of lipids and proteins, including enzymes involved in PHA metabolism [9-11]. After PHAs are extracted from cells, they are converted to the semicrystalline form and degraded by PHA depolymerases secreted from microorganisms in natural environments, such as soil, active sludge, fresh water, and seawater [12,13], the degraded compounds are converted to $\mathrm{CO}_{2}$ and $\mathrm{H}_{2} \mathrm{O}$. Thus, as the biodegradation and synthesis of PHAs are embedded in the carbon-cycle, PHAs have attracted interest as environmentally friendly materials $[14,15]$.

Many bacteria can synthesize various types of PHAs containing 3-, 4-, and 5-HA units, and more than $150 \mathrm{HAs}$ other than 3HB have been reported as constitutive units of PHAs [14]. PHAs consisting of short-chain length HAs (SCL-HAs; 3-5 carbon atoms) or medium-chain length HAs (MCL-HAs; 6-14 carbon atoms) have been detected. The former are thermoplastic in nature, whereas the latter are elastomeric. The physical and mechanical properties of PHAs can be regulated by varying the monomer composition to gain properties comparable to the petrochemical-based thermoplastics that have been used for various applications in industry, medicine, pharmacy, agriculture, and 
electronics [15]. Accordingly, PHAs have attracted industrial interest as biobased, biodegradable, biocompatible, and versatile thermoplastics $[16,17]$.

\subsubsection{Oligo- $P H B$}

Oligo-PHB was first discovered in the cytoplasmic membranes of the genetically competent bacteria Azotobacter vinelandii and Bacillus subtilis, which accumulate storage PHB [18]. Oligo-PHB was also found in Haemophilus influenza and Escherichia coli, although they do not accumulate storage PHB $[18,19]$. Oligo-PHB often forms non-covalent complexes with polyphosphate and Ca ions in vivo. Oligo-PHB-Ca-polyphosphate complexes are localized in the membrane and are responsible for DNA uptake during transformation. Furthermore, oligo-PHB-Ca-polyphosphate complexes were found in eukaryotic membranes, suggesting their possible existence in all living cells $[20,21]$. Oligo-PHB-Ca-polyphosphate complexes play a role in not only the transport of inorganic cations [22-24] but also the transport of DNA molecules [25-27].

\subsubsection{3. $c P H B$}

In 1996, cPHB was found in the membranes and the cytoplasm of E. coli cells by Huang and Reusch [28], but it is present in all organisms [29]. In E. coli, a novel type of PHB synthase $\mathrm{YdcS}$, which is different from storage PHB synthases, catalysed cPHB synthesis in collaboration with other cPHB synthases [30]. Because cPHB and oligo-PHB are ubiquitous, they are likely fundamental constituents of biological cells.

\subsubsection{PLAs}

PLAs are representative biobased plastics with good processability, transparency, biocompatibility and biodegradability. Because of these excellent characteristics, PLAs are used in packaging, containers, stationary and medical and agricultural materials [31,32]. PLAs are produced from renewable biomass through a chemo-bioprocess consisting of the fermentative production of lactic acid (LA) and chemical polymerization. LA is spontaneously polymerized by refluxing, but the molecular mass of the yielded polymer tends to be low [33]. There are several methods for synthesizing highmolecular-mass PLAs: condensation, chain elongation, and ring-opening polymerization of cyclic lactides [31]. Currently, the most common industrial method to produce PLAs is ring-opening polymerization, which is catalysed by heavy metal catalysts, typically tin $[34,35]$. However, the trace residues of heavy metal catalyst are unfavourable for certain applications, in particular, medical and food applications. Thus, replacement of the heavy metal catalyst with a safe and environmentally acceptable alternative is an important issue. To this end, one-pot production of PLA and its copolymers was achieved using 
the newly established microbial cell factory integrated by a lactatepolymerizing enzyme (LPE) (refer to section 1.2.3) [36,37].

\subsubsection{PAA}

$\beta$-Peptides are foldamers that fold into turns, helices, and sheet-like structures, analogous to the secondary structures of proteins, and show extraordinary resistance to degradation by many common peptidases and proteases $[38,39]$. Because of these properties, $\beta$-peptides have attracted increasing attention in recent years in the field of functional materials.

Thermally synthesized PAA (tPAA) is a biobased, biocompatible, biodegradable, and water-soluble polymer with high polyanionic properties [40-44]. Earlier structural analyses demonstrated that $\beta$-Asp units account for $70 \%$ of the total composition of tPAA, indicating that tPAA is composed of a high proportion of $\beta$-peptides. The analyses also showed that tPAA contains equivalent moles of D- and L-Asp units, with branching and cross-linking as unnatural structures, in addition to $\beta$-Asp units [45-49]. As unnatural structures in polymer materials generally affect the biodegradability of the material, tPAA biodegradability should be carefully considered in its applications. In addition, because polymer structure control generally results in high functionalization and performance improvement, the creation of structure-controlled PAA is one of the most preferred ways to maximize the potential of PAA as a functional material.

\subsubsection{Towards an enhanced sustainable production}

Enzymes are attractive catalysts because they are natural non-harmful catalysts that can drive reactions under mild conditions. In addition, highly specific enzymatic reactions may be capable of synthesizing polymers with fine structures from crude materials, which would reduce the cost of preparing the starting substances. This could be an advantage over chemical polymerization since the chemoprocess frequently requires extremely pure monomers, harmful catalysts and solvents, and anhydrous and high-temperature conditions.

Three main issues have hindered the widespread use of biopolymers: (1) the high production cost compared to petroleum-based polymers with similar properties; (2) the inability to produce high-performance biopolymers in substantial amounts; and (3) the difficulty in controlling the life cycle of biopolymers, i.e., control of their biodegradability and their effective chemical recycling.

To solve issues (1) and (2) for PHAs, we focused on the protein engineering of PHA synthases because PHA synthases play a central role in PHA biosynthesis, through which both the PHA production efficiency and the properties of the generated polymer can be improved [50]. We would like to highlight the current special topic of the biosynthesis of new PHA polymers incorporating 
unusual monomer units, such as LA, by PHA synthase engineering. In addition, to solve issue (3) for PHAs, we focused on the engineering of PHB depolymerases because enzymatic PHB degradation (PHB monomerization) is an important first step in the PHB recycling process. Thus, the improvement of PHB depolymerases is expected to result in the construction of an effective PHB chemical recycling system. In this chapter, we provide case studies on protein engineering of PHB depolymerase based on domain structure-based and random mutagenesis approaches. We also present additional applications using PHB depolymerases.

With respect to issues (2) and (3) for PAA, we provide the biochemical and genetic properties of PAA hydrolases. In addition, as their applications, we focus on the enzymatic synthesis of structure-controlled poly $(\alpha$-ethyl $\beta$-aspartate) ( $\beta$-PAA) by taking advantage of the substrate specificity.

\subsection{PROTEIN ENGINEERING OF PHA SYNTHASES}

\subsubsection{Enzymology and engineering of PHA synthases}

PHA synthases catalyse the polymerization of monomeric HA to polymeric PHA. The monomer substrates of PHA synthase are mainly 3HA-CoAs with various side-chain lengths, and $R$-enantiomer HA-CoAs are specifically accepted for polymerization by synthase [51]. More than 70 PHA synthases have been classified into four types based on their substrate specificities and subunit compositions (Table 1) [52].

PHA synthases belonging to type I and type II consist of single subunits (PhaC). Type I PHA synthases, represented by Ralstonia eutropha enzyme, mainly polymerize SCL-monomers (C3-C5), whereas type II PHA synthases, represented by Pseudomonas oleovorans enzyme, polymerize MCL-monomers (C6-C20). Type III PHA synthases, such as Allochromatium vinosum enzyme, consist of two hetero-subunits (PhaC and PhaE). The PhaC subunits of type III synthase are smaller than those of type I and II synthases but possess catalytic residues in the subunit. Similar to the type I synthases, these PHA synthases preferentially polymerize SCL-monomers (C3-C5). Type IV PHA synthases, such as Bacillus megaterium, are similar to type III PHA synthases in terms of possessing two subunits. However, unlike the PhaE of type III PHA synthases, a smaller protein, designated PhaR, is required to exhibit the full activity of type IV PhaC. 
Table 1. The four classes of PHA synthases

\begin{tabular}{cccc}
\hline Type & Subunit(s) & Representative species & Substrate specificity \\
\hline \multirow{2}{*}{ I } & \multirow{2}{*}{ PhaC } & $\begin{array}{c}\text { Ralstonia eutropha } \\
\text { Aeromonas caviae }\end{array}$ & C3-C5 \\
& \multirow{2}{*}{ C3-C7 } \\
\hline \multirow{2}{*}{ II } & PhaC & $\begin{array}{c}\text { Pseudomonas aeruginosa } \\
\text { Pseudomonas sp. 61-3 }\end{array}$ & C6-C14 \\
& PhaC-PhaE & Allochromatium vinosum & C3-C5 \\
\hline III & PhaC-PhaR & Bacillus megaterium & C3-C5 \\
\hline
\end{tabular}

Considering the strong relationship of the PHA synthase performance with the polymer properties, it is of great interest to alter the enzymatic properties of PHA synthase, which plays a major role in polymer synthesis. The lack of tertiary structure information for PHA synthases has limited the attempts to improve the activity and to alter the substrate specificity of these enzymes. Therefore, "irrational" methods, such as random mutagenesis and gene shuffling, have been extensively applied based on the evolutionary program $[53,54]$. In general, natural diversity provides us with attractive starting components for artificial evolution as it represents functionalized sequence spaces to a certain extent. A large population (more than 70 species) of randomly screened PHA-producing bacteria suggests that attractive prototype enzymes for molecular breeding exist. Among them, the enzyme evolution approach has been applied to type I and type II PHA synthases derived from some bacteria.

\subsubsection{Functional improvements of PHA synthases}

\subsubsection{Type I PHA synthases}

In 2001, a pioneering study that established methods for the protein engineering of PHA synthase was conducted using the best-studied enzyme, the PHA synthase of $R$. eutropha [55]. The in vitro evolutionary program was first constructed by coupling an error-prone PCR-mediated mutagenesis with a plate-based, high-throughput screening method to create mutants with acquired beneficial functions [55]. A mutant library of the R. eutropha PHA synthase gene was prepared through the colony formation of transformed cells of Escherichia coli. There was a good correlation between the change in PHB accumulation caused by the various mutations in the $R$. eutropha PHA synthase gene and the change in the enzymatic activity of the mutants. Multi-step mutations, including an activity loss and intragenic suppression-type activity reversion, effectively produced mutants with increased activity [56]. The mutant enzymes were identified by mutation sequence analysis, and a second round of mutation was used to evolve these enzymes to proteins with better characteristics than the wild-type enzyme. Through this intragenic suppression-type mutagenesis, 2.4-fold specific activity towards 3HB-CoA 
compared to the wild-type enzyme was acquired by the mutation of Phe420Ser (F420S) in an intrinsically highly active type I PHA synthase [56].

In another case, a screened beneficial mutation, Gly4Asp (G4D), exhibited higher levels of protein accumulation and PHB production than the recombinant E. coli strain harbouring the wild-type PHA synthase [57]. For intragenic suppression-type mutagenesis, second-site reversion was dependent or independent of primary mutation in the activity. Secondary mutation of F420S and G4D were independent of the primary mutation. Subsequently, site-specific saturation mutagenesis was also performed on the codon encoding the G4 residue of the PHA synthase of $R$. eutropha, and many substitutions resulted in much higher PHB content and higher molecular mass polymers [58].

Aeromonas caviae (punctata) PHA synthase is unique among the type I PHA synthases since it can synthesize not only PHB homopolymer but also random copolyesters of 3HB and 3-hydroxyhexanoate (3HHx). Kichise et al. performed the first successful in vitro molecular evolution experiments on PHA synthase from A. caviae by targeting to the limited region of the enzyme [59]. Two evolvants exhibited increased specific activities towards 3HB-CoA of $56 \%$ and $21 \%$ compared to the wild-type enzyme. These mutations led to enhanced accumulation (up to 6.5-fold that of the wild-type enzyme) of $\mathrm{P}(3 \mathrm{HB}-c o-3 \mathrm{HHx})$ and increases in the $3 \mathrm{HHx}$ molar fraction (16-18 mol\% compared to $10 \mathrm{~mol} \%$ of the wild-type PHA synthase) in recombinant E. coli strains grown on dodecanoate. A combination of these beneficial mutations (N149S/D171G) synergistically altered the enzymatic properties, leading to the synthesis of PHA copolymers with enhanced 3HA fractions and increased molecular mass from the recombinant $R$. eutropha [60]. A Japanese chemical company, Kaneka Co. Ltd., has initiated the industrial production of this type of polyester, named AONILEX, in 2011. The commercial-based manufacturing of this polymer product involves the above-mentioned evolved enzymes. In a separate study, A. caviae PHA synthase was engineered in vivo using the mutation-prone strain E. coli, which has a 5,000-fold higher mutation rate than wild-type E. coli, and the mutants were screened for enhanced PHB accumulation in recombinant E. coli [61]. Additionally, the mutants synthesized PHAs with increased molecular mass, but in contrast to the previous study, the $3 \mathrm{HHx}$ fraction was only slightly different from the wild-type composition.

A junction site for the interconnection of heterogeneous enzymes based on the predicted secondary structures allowed chimera-genesis of the PHA synthase from $R$ e eutropha with the partner PHA synthase from A. caviae. The chimeric mutant exhibited higher activity and expanded substrate specificity compared to the original enzymes [62]. For PHA synthases, directed evolution studies have advanced from random approaches to much more systematic approaches, such as chimera-genesis, recombination and shuffling. 


\subsubsection{Type II PHA synthases}

In contrast with type I PHA synthases, type II PHA synthases typically have substrate specificity towards MCL-3HA-CoA substrates and relatively poor substrate specificity towards SCL-3HA-CoA substrates, such as 3HB-CoA. An exception to this is the type II PHA synthase of Pseudomonas sp. 61-3, which has significant substrate specificity towards 3HB-CoA (Table 1). In the landmark study of Takase et al., the in vitro evolutionary technique was applied to PhaC1 PHA synthase from Pseudomonas sp. 61-3 to increase the activity towards 3HB-CoA monomers [63]. Substitutions at two amino acid residues, Ser325 and Gln481, were found to dramatically affect the production of PHB homopolymer in recombinant E. coli using glucose as the carbon source. The codons for these amino acids were subjected to site-specific saturation mutagenesis, and several individual substitutions were found to dramatically increase the level of PHB production. These mutations were combined as double mutants to further increase the level of PHB production (340-400-fold that of the wild-type enzyme) [63]. The changes in the in vivo-produced $\mathrm{P}$ (3HB-co-3HA) copolymer molar compositions correlated well with the in vitro biochemical data of the substrate specificity and activity of the enzymes and represents one of the most complete studies to date [64].

The findings of these studies for type II PHA synthase are useful for evaluating similar evolution strategies of other types of PHA synthases based on their amino acid sequence alignment. For example, position 481 in PhaC1 PHA synthase from Pseudomonas sp. 61-3 was found to be one of the residues that determined the substrate specificity of the enzyme, as described above. Interestingly, the amino acid residues corresponding to the position of this enzyme are conserved within each type of PHA synthase: Ala for type I, Gln for type II, Gly for type III and Ser for type IV enzymes. Thus, the effects of mutating the highly conserved alanine (Ala510) of the R. eutropha PHA synthase (position 481 in Pseudomonas sp. 61-3 PhaC1) were analysed via site-specific saturation mutagenesis. Mutations at Ala510 affected the substrate specificity of the $R$. eutropha PHA synthase, allowing slightly higher 3HA incorporation than the wild-type PHA synthase in $R$. eutropha PHB-4 (PHA negative mutant) [65].

Two additional beneficial positions, Glu130 [66] and Ser477 [67], were identified through the in vitro evolution screening. As illustrated in Figure 2 (A) and (B), "mutation scrambling" among four beneficial positions (130, 325, 477,481 ) to increase activity, change the substrate specificity, and regulate the polymer molecular mass would further create new super-enzymes. A possible mechanistic model for PHA polymerization was recently proposed on the basis of the accumulated evolutionary studies [68]. Furthermore, the useful evolvants obtained through systematic enzyme evolution have been supplied to other organisms, including plants $[69,70]$. The impacts of these reports 
inspired additional research groups to apply directed evolution to individual PHA synthases of interests [71-73].
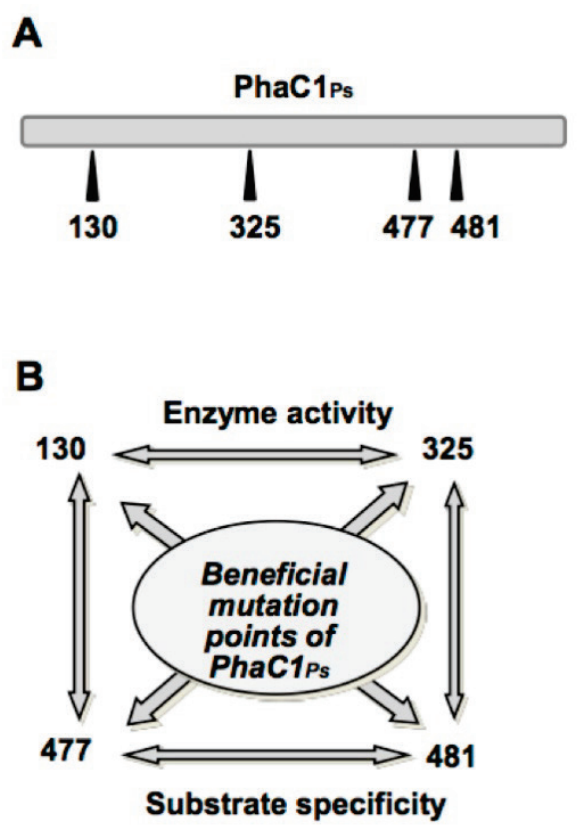

Figure 2. Functional mapping of beneficial positions (A) and relationships among the residues related to enzymatic activity and substrate specificity of PHA synthase from Pseudomonas sp. 61-3 (B)

\subsubsection{Creation of lactate-polymerizing enzyme (LPE)}

The pioneering work on the exploration of LA-polymerizing activity by PHA synthases was reported by Valentin et al. [74], in which PLA biosynthesis was performed by monitoring the activity of PHA synthases towards synthetic LA-CoAs ( $R$ and $S$ enantiomers). Several PHA synthases were evaluated for LA-polymerizing activity, and a class III PHA synthase from Allochromatium vinosum exhibited weak CoA-releasing activity [74]. In a similar report, Yuan etal. reported the activity of $A$. vinosum PHA synthase towards $(R)$-LA-CoA [75]. Unfortunately, in both cases, polymerization was not observed or was negligible, suggesting that PHA synthase could hydrolyse CoA ester to release CoA but could not progress further through polymerization to form a polymer.

In this context, Taguchi et al. formally reported the first prototype LPE in 2008 as a PHA synthase with acquired LA-polymerizing activity through in vitro and in vivo experiments [36]. The first indication of LA-polymerizing activity was 
obtained through a water-organic solvent two-phase in vitro system [36,37]. The activity towards LA-CoA was tested in the absence and presence of 3HBCoA using representative PHA synthases belonging to the four classes of natural PHA synthases, together with three engineered ( $\mathrm{PhaC} 1_{\mathrm{Ps}}$ mutants) from Pseudomonas sp. 61-3. The engineered PHA synthases were two single mutants [Ser325Thr (ST) and Gln481Lys (QK)] and one double mutant carrying both mutations (STQK). The two mutants were selected based on their improved activity and/or broader substrate specificity [53,54]. The natural synthases and mutants did not form a clear-polymer with LA-CoA alone but did with 3HB-CoA. However, when LA-CoA was supplied together with 3HB-CoA, one mutant, PhaC1 $1_{\mathrm{Ps}}(\mathrm{STQK})$, exhibited polymer-like precipitation. Analysis of the precipitant revealed that it consisted of $36 \mathrm{~mol} \%$ of the LA unit. This was the first report of a PHA synthase with the ability to incorporate an LA unit to form $\mathrm{P}(\mathrm{LA}-\mathrm{co}-3 \mathrm{HB})$. The finding that PhaC1 $1_{\mathrm{Ps}}(\mathrm{STQK})$ could polymerize LA was a demonstration of evolutionary engineering as a powerful tool to generate biocatalysts with desired properties. By demonstrating the in vitro activity of $\mathrm{PhaC}_{\mathrm{Ps}}(\mathrm{STQK})$ towards LA-CoA, it was presumed that heterologous expression of this LPE could result in in vivo synthesis of LA-based polyesters, thus creating microbial factories for LA-based polyester synthesis. The metabolic engineering approach outlined in this contribution provides an entirely new single organism generation of the polymer, a major breakthrough in the field. This finding opens the door to optimization of this type of process to become cost competitive with other commodity polymers.

In a subsequent study, based on the improved activity of a point mutation at position 420 (F420S) of a type I PHA synthase $\left(\mathrm{PhaC}_{\mathrm{Re}}\right)$ from $R$. eutropha [56], the same mutation was introduced into the ancestral LPE [PhaC1 $1_{\mathrm{Ps}}(\mathrm{STQK})$ from Pseudomonas sp. 61-3] to create a triple mutant of LPE with S325T, Q481K and the new mutation F392S, which corresponds to F420S of $\mathrm{PhaC}_{\mathrm{Re}}$ [76]. When the new LPE [PhaC1 $1_{\mathrm{Ps}}$ (STQKFS)] was expressed in E. coli, a copolymer with $45 \mathrm{~mol} \% \mathrm{LA}$ and a polymer content of $62 \mathrm{wt} \%$ was synthesized, in comparison with $\mathrm{P}(26 \mathrm{~mol} \% \mathrm{LA}-\mathrm{co}-3 \mathrm{HB})$ obtained with the prototype LPE, PhaC1 ${ }_{\mathrm{Ps}}(\mathrm{STQK})$, under aerobic culture conditions. Additionally, the cells harbouring $\mathrm{PhaC} 1_{\mathrm{Ps}}$ (STQKFS) synthesized P(LA-co-3HB) with $62 \mathrm{~mol} \% \mathrm{LA}$ and a polymer content of $12 \mathrm{wt} \%$. During the same study, saturation mutagenesis of LPE at the same site (position 392) yielded mutants that gave varying LA fractions in the copolymers; however, F392S was superior to the other mutants in incorporating LA. This study demonstrated the effectiveness of the enzyme engineering of LPE in two directions: improved LA incorporation and polymer yield for both aerobic and anaerobic culture conditions [76]. Furthermore, copolymers incorporating 2-hydroxy acids (2HAs), such as 2-hydroxybutyrate $[77,78]$ and glycolate $[79,80]$, may lead to copolymers with novel properties, including biodegradability (Figure 3). This expansion of PHA synthase to 2HA-polymerizing enzymes has prompted us to create additional enzymes with 
acquired activity towards new and unusual monomer substrates, resulting in new polymers.

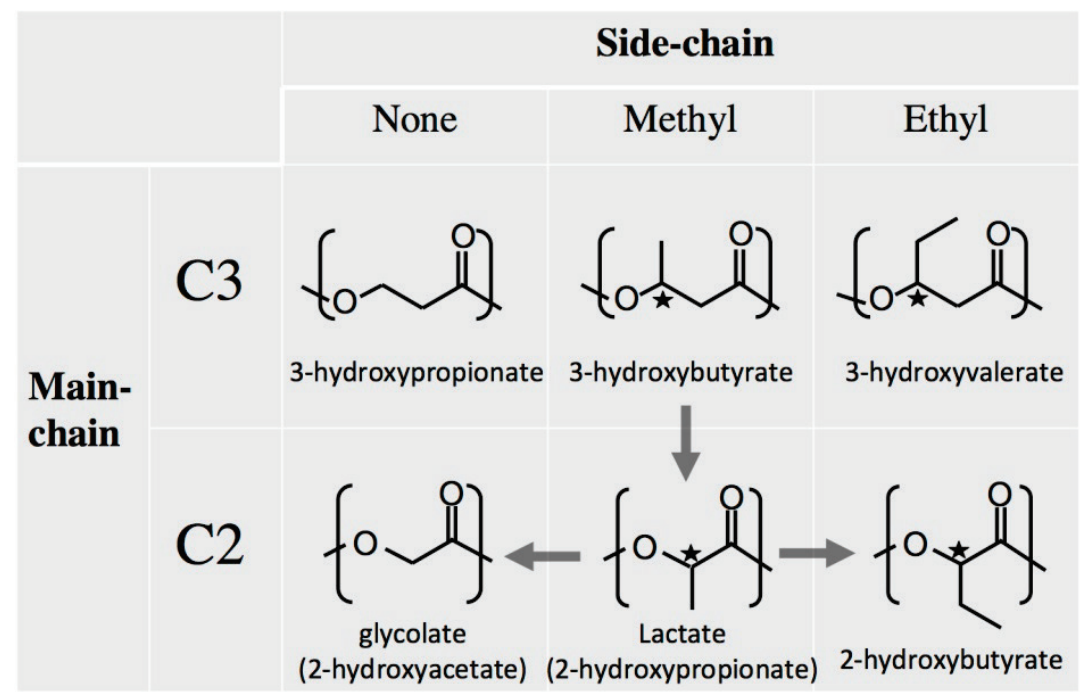

Figure 3. Structural diversities in main-chain and side-chain of PHA back bone which can be recognized by natural and artificially evolved PHA synthases. Asterisks indicate the chiral center in monomer units of PHA.

Regarding the reports on LPE, several similar studies have been followed using LPE homologous enzymes [81,82]. The best-studied PHA synthase from $R$. eutropha has been successfully engineered to LPE by a single mutation at a beneficial position corresponding to position 481 of Pseudomonas sp. 61-3 PHA synthase [83]. This implies functional compatibility between PHA members for acquiring LPE activity. Therefore, advanced types of LPE could be obtained by artificial evolution of the prototype LPE and by exploration of natural PHA synthases that potentially possess new substrate specificities, such as LA-polymerizing activity $[84,85]$. 


\subsection{PROTEIN ENGINEERING OF PHB DEPOLYMERASE}

\subsubsection{Biochemical and genetic properties of PHB depolymerases}

After the use and disposal of PHA materials, they are decomposed to oligomers and/or monomers by extracellular PHA depolymerases with degradation ability for crystalline PHAs. More than 30 PHA depolymerase genes, with experimentally verified activity, have been identified, and the PHA depolymerase Engineering Database has been established based on their sequence similarity [86]. Among the PHA depolymerases, multi-domain PHB depolymerases have been extensively studied [87-89]. Multi-domain PHB depolymerases generally consist of a catalytic domain (CD) at the N-terminus, a substrate-binding domain (SBD) at the C-terminus, and a linker region connecting the two domains [87]. Genetic analysis shows that PHB depolymerases contain a lipase box pentapeptide [Gly-Xaa1-Ser-Xaa2-Gly] as an active residue, indicating that these enzymes are serine hydrolases. The enzymatic degradation of PHB by the multi-domain PHB depolymerases is considered to proceed via a two-step reaction at the solid-liquid interface, as shown in Figure 4. The PHB depolymerase approaches and adheres to the PHB surface via SBD, followed by hydrolysis of the polymer chain by CD. Thus, elucidation of the mechanisms of enzyme adsorption and enzymatic hydrolysis may provide strategies for the design of new PHB polymer materials with the desired environmental stability and biodegradability. Furthermore, the improvement of PHB depolymerases could result in effective enzymatic recycling of $\mathrm{PHB}$ materials. To improve PHB depolymerases, it is important to understand the mechanisms underlying which and how amino acid residues participate in the adsorption and hydrolysis processes. Thus, we have

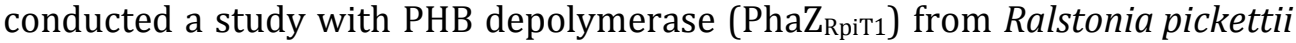
$\mathrm{T} 1$, which is one of the best-studied PHB depolymerases.

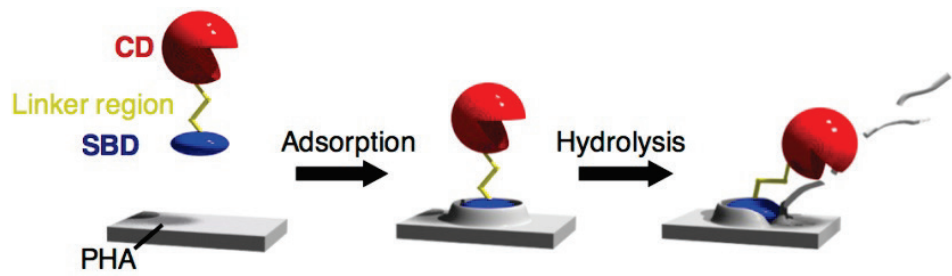

Figure 4. Schematic illustration of the enzymatic degradation of PHA by PHB depolymerase 


\subsubsection{Protein engineering of the $S B D$ region of $P h a Z_{R p i T 1}$}

The structural aspects of an enzyme generally provide crucial information about the interaction between the enzyme and its ligand. However, because of the paucity of information about the 3D structures of multi-domain PHB depolymerases, there are few insights into which and how the amino acid residues in their SBD are involved in the enzyme adsorption to the PHB surface.

Directed evolution approaches are frequently used to explore, manipulate, and optimize enzyme properties, including substrate specificity, catalytic activity, thermostability, and solubility, because no information about the tertiary structure of the enzyme is required [90-93]. These approaches are also applicable to the analysis of mutation effects on enzyme activity to provide useful information for the improvement of enzyme function. To clarify which and how residues are involved in the adsorption, we investigated the interaction between PhaZ $\mathrm{RpiT1}_{\text {and }}$ an PHB surface using a combination of PCR random mutagenesis targeted to only SBD and an in vivo screening system, as shown in Figure 5 (A) [94]. In the analysis of recombinants with low PHB-degrading activity, Ser410, Tyr412, Val415, Tyr428, Ser432, Leu441, Tyr443, Ser445, Ala448, Tyr455, and Val457 were replaced with different residues with hydropathy indices opposite to theirs at high frequency [Figure 5 (B)]. The results suggested that PhaZ $Z_{\text {RiT1 }}$ adsorbs to the PHB surface via the formation of hydrogen bonds between hydroxyl groups of the residues and carbonyl groups in the PHB polymer and via hydrophobic interaction between the hydrophobic residues and the methyl groups in the PHB polymer.

Among these positions, Leu441, Tyr443, and Ser445 were predicted to form a $\beta$-sheet structure to orient in the same direction and to possibly interact with the PHB surface. Among the amino acid substitutions found at these residues, the hydropathy indices of the L441H (replacement of Leu441 with His), Y443H (replacement of Tyr443 with His), and S445C (replacement of Ser445 with Cys) mutations dramatically changed. Functional analyses of the $\mathrm{L} 441 \mathrm{H}$, $\mathrm{Y} 443 \mathrm{H}$, and $\mathrm{S} 445 \mathrm{C}$ enzymes indicated that these mutations had no influence on their structures and their ability to cleave the ester bond, while their PHB-degrading activity differed from that of the wild-type [95]. Kinetic analysis of PHB degradation by the mutants suggested that the hydrophobic residues at these positions are important for enzyme adsorption to the PHB surface and may more effectively disrupt the PHB surface to enhance the hydrolysis of PHB polymer chains than the wild-type enzyme. Further, surface plasmon resonance (SPR) analysis revealed that these substitutions altered the association phase rather than the dissociation phase in the enzyme adsorption to the polymer surface. 


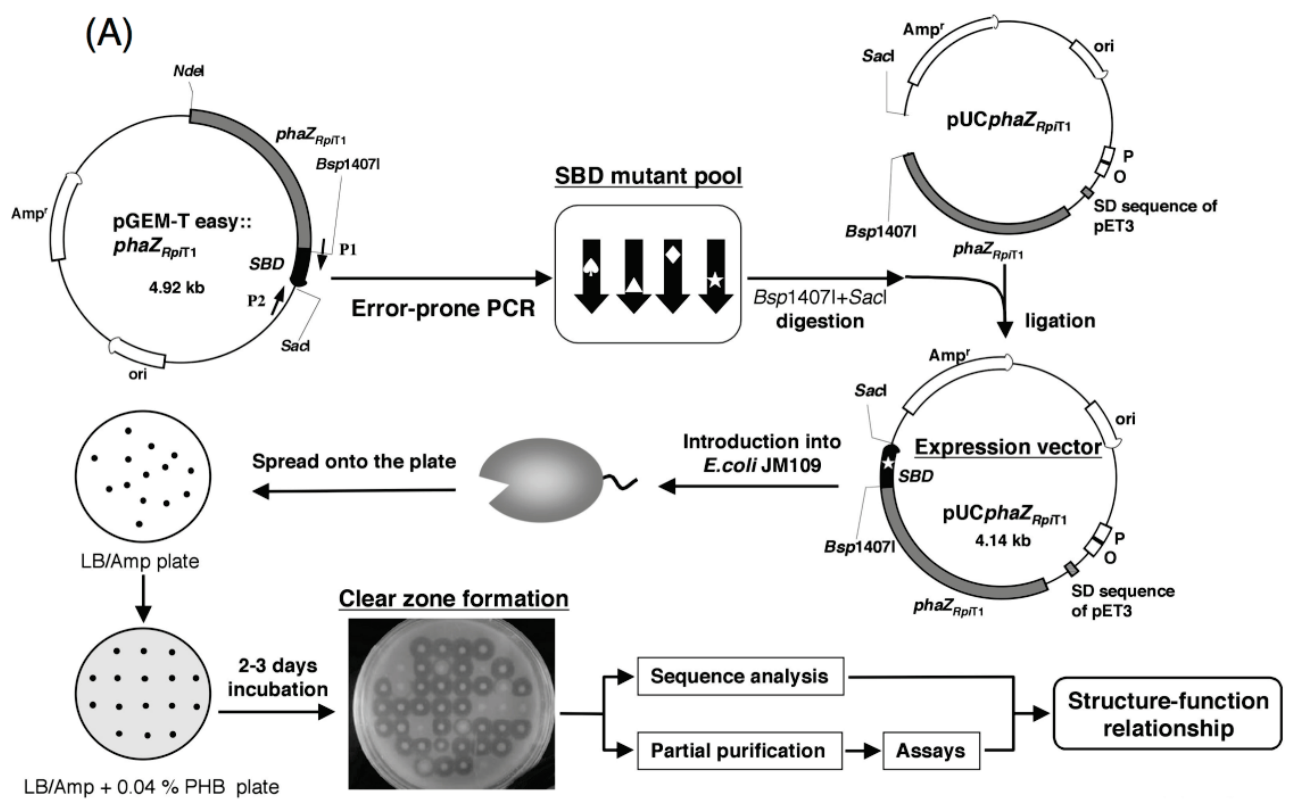

(B)

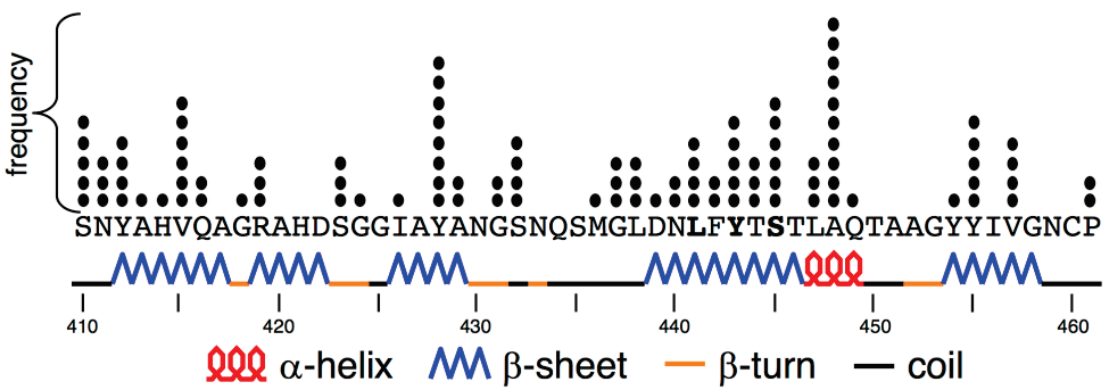

Figure 5. (A) In vivo assay system for assessment of mutational effects of the substrate-binding domain of PhaZ $\mathrm{R}_{\mathrm{Rpi} 1}$ on PHB degradation. Schematic flow diagram of the system is illustrated. This system is composed of PCR-mediated random mutagenesis in the substrate-binding domain region of $\mathrm{PhaZ}_{\mathrm{RpiT} 1}$ gene, preparation of mutant library, primary plate assay of PHB degradation (clear-zone formation), nucleotide sequencing and PHB degrading and adsorbing assays of partially purified mutant enzymes. (B) Positions and frequencies of PCR-mediated single mutations in the region coding for $\mathrm{SBD}$ of $\mathrm{PhaZ}_{\mathrm{RpiT1}}$, together with its predicted secondary structure.

Recently, the crystal structures of single-domain PHB depolymerases from Penicillium funiculosum (PhaZ $\mathrm{Pfu}_{\mathrm{P}}$ ) and PhaZ7 from Paucimonas lemoignei (PhaZ7 ${ }_{\mathrm{Ple}}$ ) were determined [96-98]. Their structural analyses suggested that Tyr and/or hydrophobic residues are involved in the enzyme adsorption to the PHB surface. Taken together, PHB binding ability of PhaZ $Z_{\text {RpiT1 }}$ can be improved by substituting a hydrophilic residue with a hydrophobic one at the positions 
of 441, 443 and 445 . Tyr at position 443 was targeted for substitution with a more hydrophobic residue because its hydrophobicity is medium to high compared to those of general naturally occurring amino acid residues [99]. In the design of a mutant enzyme with an amino acid substitution at this position, the following factors were taken into consideration: (1) to achieve higher hydrophobicity than the original residue, (2) to retain the $\beta$-sheet structure, and (3) to minimize the change to the volume of the amino acid residue after the substitution. The substitution of Tyr443 with Phe (Y443F) was considered to be appropriate. Analysis of the Y443F enzyme indicated that the mutation had no influence on the structure and the ester bond cleavage activity, while this mutant had higher PHB degradation activity than the wild-type. Thus, this finding supports the above assumptions and indicates the importance of hydrophobic interaction for PHB adsorption of PhaZ $Z_{\text {RpiT1. }}$.

\subsubsection{Protein engineering of the $C D$ region of $P h a Z_{R p i T 1}$}

Cell surface display is a valuable technique for peptide/protein expression on the surface of microorganisms by fusion with the appropriate anchoring motifs [100]. The cell surface display of functional and useful peptides/proteins has become a useful strategy in various applications, such as whole-cell biocatalysts and bioabsorbents, live vaccine development, antibody production, and peptide library screening. This method is also useful for enzyme library screening because the displayed protein is accessible to the external environment and is thus able to interact with substrates easily, enabling the screening of large libraries [101].

There is also little knowledge about the improvement of the CD function of

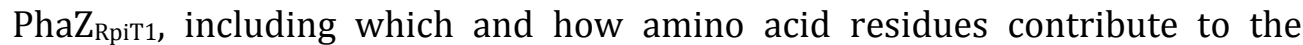
hydrolytic activity. Thus, the $\mathrm{CD}$ of $\mathrm{PhaZ}_{\mathrm{RpiT1}}$ was targeted for directed evolution using random mutagenesis and DNA recombination to enhance its ester bond cleavage ability (Figure 6) [102]. To easily react with water-soluble substrates, such as $p$-nitrophenyl butyrate (pNPC4), we used a cell surface display system to create a mutant library [103]. Clones displaying mutant enzymes with a 4- to 8-fold increase in pNPC4 hydrolysis activity were obtained, in comparison with those displaying the wild-type. Gene analysis showed that eight of the improved mutant enzymes contained N285D or $\mathrm{N} 285 \mathrm{Y}$ mutations. As beneficial mutations are accumulated and deleterious mutations are simultaneously removed from the improved mutants through DNA recombination [104], the N285D and N285Y mutations are probably beneficial for pNPC4 hydrolysis. Kinetic studies revealed that the increase in catalytic efficiency for pNPC4 hydrolysis by the mutant enzymes is attributed to the high $V_{\max }$ values. In contrast to pNPC4 hydrolysis, the PHB degradation rates by $\mathrm{N} 285 \mathrm{D}$ and $\mathrm{N} 285 \mathrm{Y}$ were slower than that of the wild-type enzyme, indicating that these mutations are unfavourable for PHB degradation. The kinetics of PHB degradation demonstrated that the N285D and N285Y mutations lowered the hydrolysis activity for the PHB polymer chain 
compared to the wild-type enzyme, despite retention of the binding activity for the PHB polymer surface. To exhaustively examine the effects of mutations at position 285, we conducted site-directed saturation mutagenesis at that position, and the resultant mutant enzymes (N285X) were evaluated for $p$-nitrophenyl ester (pNPCn) hydrolysis and PHB degradation [105]. The kinetic studies demonstrated that PHB-degrading activities of N285X were reciprocally related to their pNPCn-hydrolysing activities and that $\mathrm{N} 285 \mathrm{H}$ showed comparable activity of PHB degradation to wild-type enzyme. In the PHA depolymerase engineering database, $\mathrm{PhaZ}_{\text {RpiT1 }}$ belongs to the e-dPHAscl homologous family 8, and Asn285 in PhaZ $\mathrm{RpiT1}_{1}$ is highly conserved in most PHB depolymerases in this family. However, for PHB depolymerase from Burkholderia thailandensis E264, the amino acid residue corresponding to

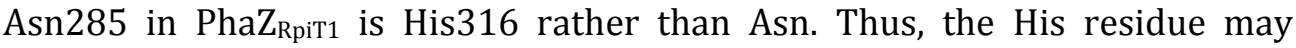
functionally substitute for Asn at that position on PHB degradation in the course of the molecular evolution of PHB depolymerases in nature.

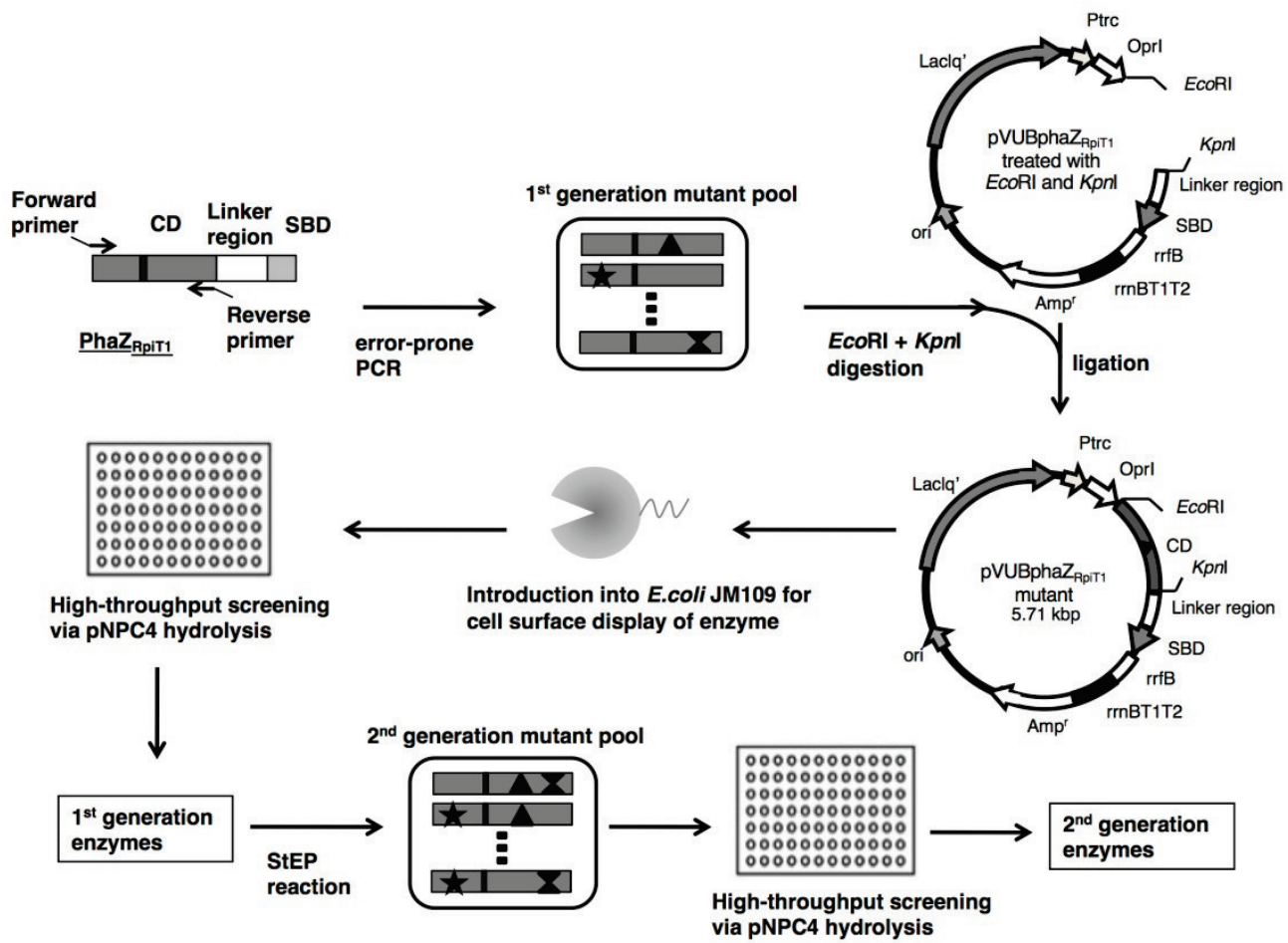

Figure 6. Directed evolution targeted at the catalytic domain (CD) of PhaZ $\mathrm{R}_{\text {RiT1 }}$ using the in vivo screening system in the cell surface display system. A schematic diagram of the mutational effects analysis is illustrated. This system consists of random mutagenesis by error-prone PCR in the CD of PhaZ $Z_{\text {RpiT1, }}$ cell surface display of enzyme, high-throughput microplate screening via p-nitrophenyl butyrate (pNPC4) hydrolysis, staggered extension process (StEP), and nucleotide sequencing. 


\subsubsection{Proposed active site model of $P_{\text {haZ }}$ RiT1}

The correct orientation of a PHB polymer chain to the active site is necessary to realize effective PHB degradation by PHB depolymerase. Bachmann and Seebach proposed that PhaZ $Z_{\text {RiT1 }}$ has four subsites $(2,1,-1$, and -2$)$ in its active site, in which three of the subsites must be occupied by 3HB units for cleavage to occur at the centre of the active site [106]. Homology modelling of PhaZ $\mathrm{Z}_{\text {RiT1 }}$ (the modelled residue range was from positions 124 to 294) based on the crystal structure of PhaZ $Z_{\mathrm{Pfu}}$ suggested that Asn285 (color-coded according to molecular species) of PhaZ $\mathrm{RpiT}_{\text {1T }}$ is located at the mouth of the crevice, immediately above His273, which probably involved subsite -1 (Figure 7 (A)). Asn285 was positioned as if to cover subsite -1 and to inhibit substrate access. Taking the homology modelling results and the aforementioned findings on the cleavage mechanism into consideration, we propose a simple schematic model for PhaZ $Z_{\text {ppiT1, }}$ as shown in Figure 7 (B). In this model, Ser139 participates in the nucleophilic attack of the carbonyl carbon of a PHB chain, and its nucleophilicity is enhanced by the His273-Asp214 hydrogen bonding system. The residue Asn285 is positioned relatively close to His273 located in subsite -1 as if to cover the subsite. The location of Asn285 in the subsite probably leads to the regulation of the recognition of substrate molecules, such as pNPC4 and the PHB polymer chain, possibly indirectly via conformational change. Similar findings have been reported in lipases and PhaZ7 $7_{\text {Ple, }}$ where activation via conformational change is required to uncover the active site $[107,108]$.

\subsubsection{Applications of PHB depolymerases}

Recently, in vitro enzymatic synthesis has become an effective method for the synthesis of environmentally acceptable biopolymers. The majority of enzymes studied for polymerization are hydrolases, most notably lipases and proteases, for both ester and amide bond formation [109]. Although PHB depolymerases have no lipase activity, they have a catalytic centre resembling that of lipase, making them possible catalysts for esterification and PHB polymerization. PHB depolymerases can be employed for the production of esters in anhydrous media [110-113]. Similarly, PHA depolymerases from Pseudomonas fluorescens GK13 and Streptomyces venezuelae S01 were applied to esterification and PHB synthesis $[114,115]$.

An additional application, surface patterning using biopolymers and their depolymerases, is included here. The patterning of polymeric and organic surfaces has been widely investigated for the fabrication of soft interfaces [116], and micro-contact printing $(\mu \mathrm{Cp})$ and scanning probe microscopy-based lithography have been used as the surface patterning methods. Recently, the development of enzyme-catalysed surface patterning has become one of the most important and attractive challenges for site-selective patterning with various surface morphologies $[117,118]$. Thus, we prepared biopolymer 
surfaces with different enzymatic degradability using simple surface modification by UV-ozone treatment/UV irradiation, followed by controlled enzymatic degradation, resulting in regular surface patterning [119]. Lee et al. reported the specific patterning of proteins on the PHA surface by $\mu \mathrm{Cp}$. Their method was based on the site-selective binding of SBD to PHA, which enables the micropatterning of proteins/DNA fused to the SBD on a PHA thin film [120-122].

(A)

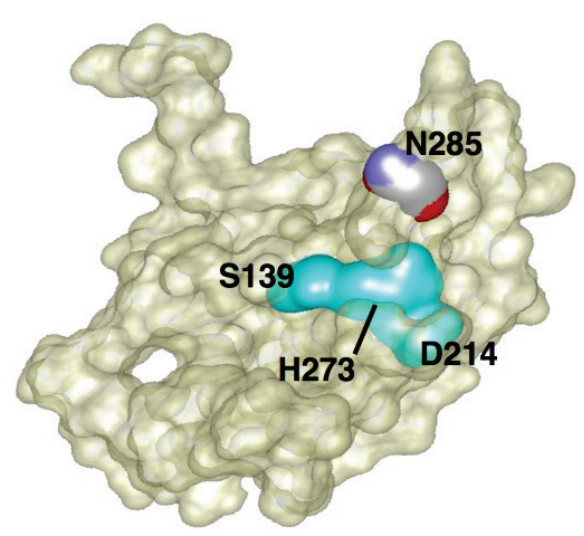

(B)
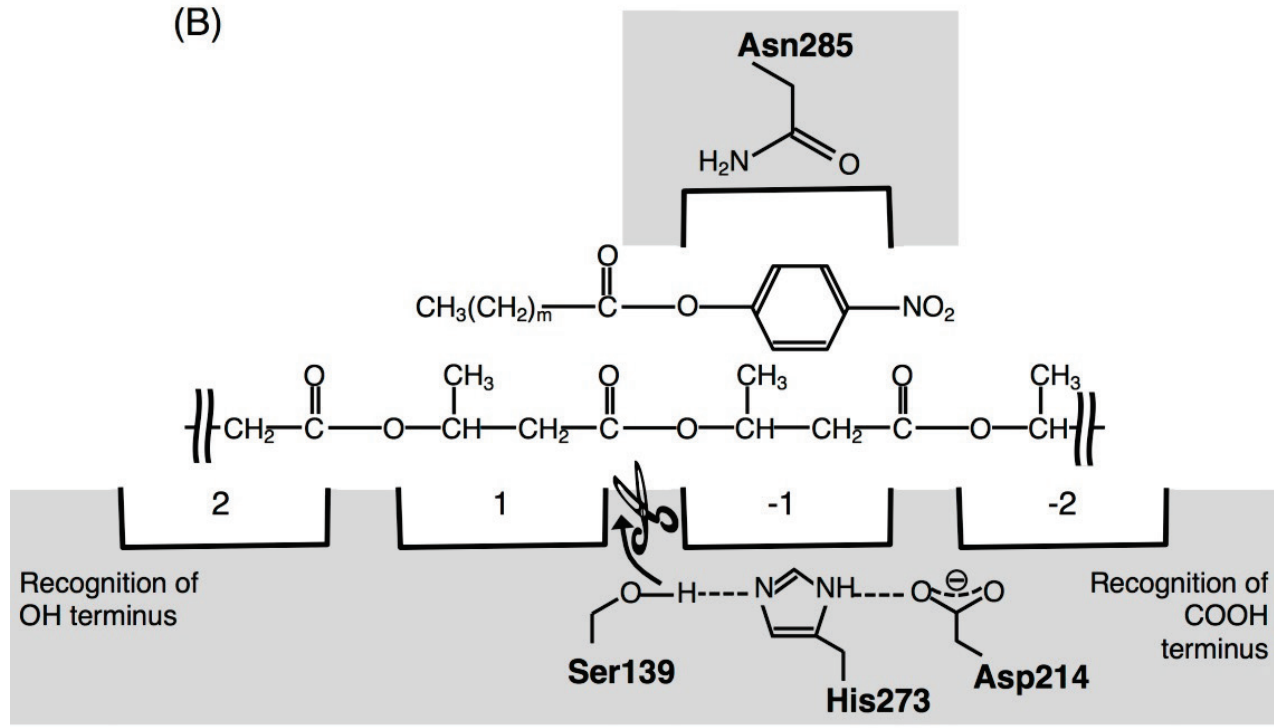

Figure 7. (A) Molecular surface representation of the homology model of PhaZ RpiT1. The positions of catalytic triad residues (S139, D214, and H273) (cyan), as well as residue N285 (color-coded according to molecular species) are indicated. (B) Newly proposed schematic model of the active site in the CD of PhaZ $Z_{\text {RpiT1 }}$. 


\subsection{PAA HYDROLASES}

\subsubsection{Biochemical and genetic properties of PAA hydrolases}

tPAA biodegradability has been assessed using semicontinuous activated sludge (SCAS), mini-continuous activated sludge (Mini-CAS), and modified sturm $\left(\mathrm{CO}_{2}\right.$ production) tests $[40,42]$. Although microorganisms and enzymes are directly responsible for the biodegradation and lifetime control of tPAA, there were no reports of tPAA biodegradation by isolated microorganisms and enzymes until our research group's work [123,124]. Sphingomonas sp. KT-1 and Pedobacter sp. KP-2 were isolated as tPAA-degrading and tPAA-assimilating microorganisms from river water $[125,126]$. The former strain took up low molecular weight tPAA and completely degraded it to Asp monomers. In contrast, the latter strain extracellularly degraded tPAA to oligomers, followed by the intracellular complete degradation of the oligomers. The enzymes related to tPAA metabolism (PAA hydrolases-1 and -2) were purified from the soluble fraction of these bacteria [127-129]. Their biochemical, structural, and genetic properties are listed in Table 2. PAA hydrolase-1 from Sphingomonas sp. KT-1 (PahZ1 $1_{\text {KT-1) }}$ is a single-domain enzyme (ca. $30 \mathrm{kDa}$ ) and contains a lipase box as its catalytic centre, indicative of a Ser-type hydrolase [130]. PahZ1 $1_{\mathrm{KT}-1}$ catalyses the cleavage of the amide bonds between $\beta$-Asp units in tPAA to generate oligo(Asp)s (OAA) via an endotype process [131]. This reaction is followed by the exo-mode hydrolysis of OAA by another PAA hydrolase, PAA hydrolase-2 (PahZ2 ${ }_{\mathrm{KT}-1)}$. Gene analysis showed that PahZ2 $2_{\mathrm{KT}-1}$ is similar to exo-type carboxypeptidase G2 and the putative metallopeptidase from Caulobacter crescentus CB15. As PahZ1 $1_{\text {KT- } 1}$ and PahZ2 кт-1 $_{1}$ were purified from the soluble fraction and their deduced amino acid sequences had signal peptide sequences, they probably participate in tPAA degradation in the periplasmic space, as shown in Figure 8 [123].

PAA hydrolase-1 from Pedobacter sp. KP-2 (PahZ1 $1_{\mathrm{KP}-2}$ ) was found to be localized in the periplasm fraction, which was supported by the existence of a signal peptide sequence in its deduced amino acid [129]. PahZ1 $1_{\mathrm{KP}-2}$ is also a single-domain enzyme ( $c a .31 \mathrm{kDa}$ ) and a Ser-type hydrolase, similar to PahZ1 $_{\mathrm{KT}-1 .}$ GPC and NMR analyses of the products during tPAA hydrolysis by PahZ1 $1_{\mathrm{KP}-2}$ demonstrated that this enzyme specifically, but not completely, cleaves the amide bond between $\beta$-Asp units in tPAA via an endo-type process. Because the tPAA molecule contains equivalent moles of D- and L-Asp units, uncommon sequences in nature [(D-Asp)-(D-Asp), (D-Asp)-(L-Asp), (L-Asp)-(D-Asp)], in addition to (L-Asp)-(L-Asp), may be formed, which may affect the

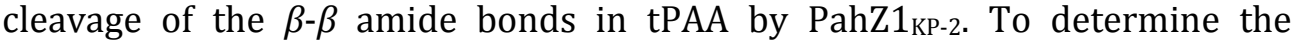
mechanism, we performed the hydrolysis of $\beta$-tri(Asp)s with all possible combinations of L- and D-Asp units by PahZ1 $1_{\mathrm{KP}-2}[124,132]$. The results suggested the following structural features of $\mathrm{PahZ} 1_{\mathrm{KP}-2}$ at its substrate-binding site: (1) the active site contains four subsites $(2,1,-1$, and -2$)$, three of which 
Table 2. tPAA- and $\beta$-peptide-degrading microorganisms and their hydrolases

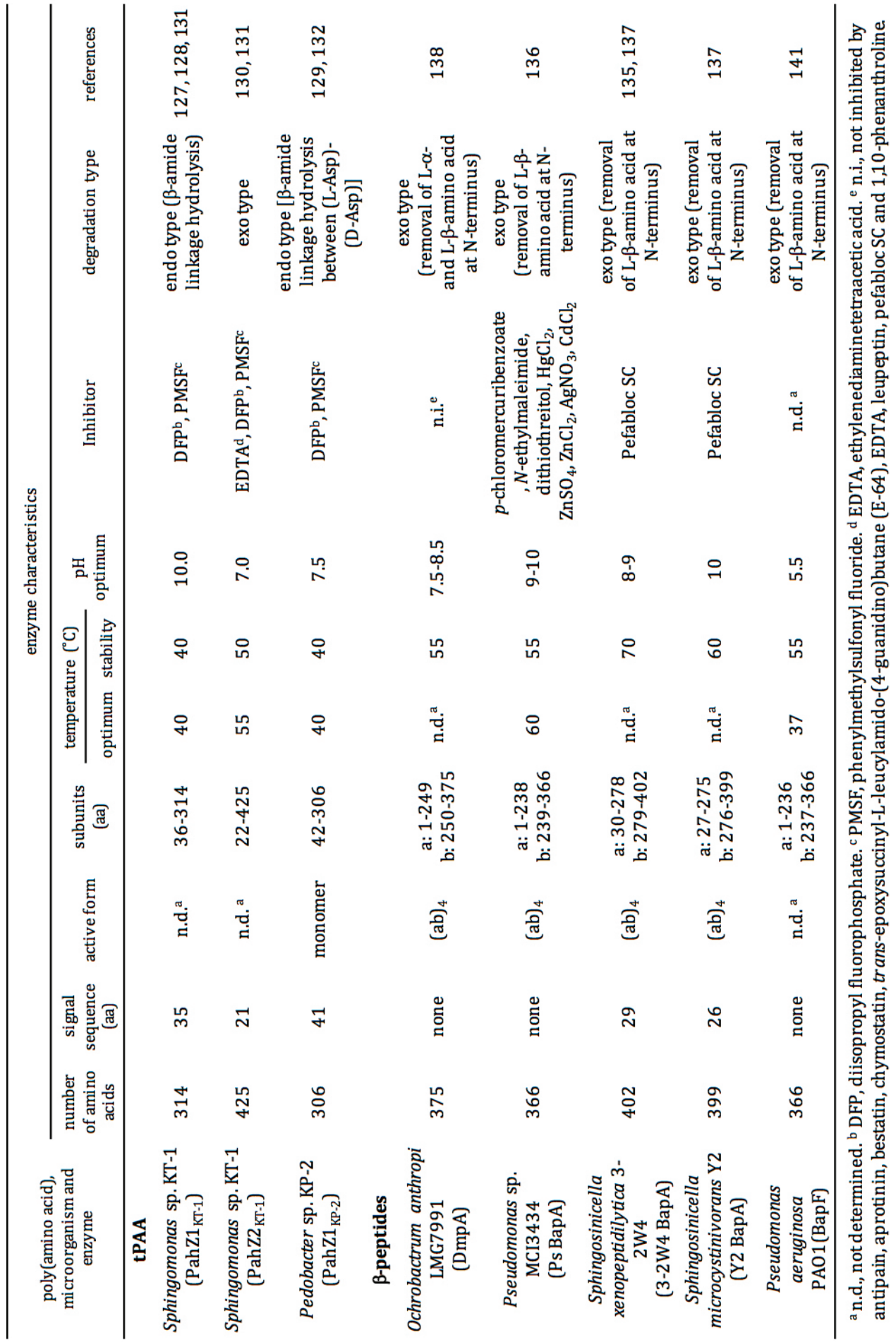


need to be occupied by Asp units for cleavage to occur; (2) for the hydrolysis to proceed, subsite 1 must be occupied by an L-Asp unit, whereas the other three subsites accept either L- or D-Asp units; (3) for the two central subsites between which cleavage occurs, the (L-Asp)-(D-Asp) sequence is the most favourable for cleavage (Figure 9).

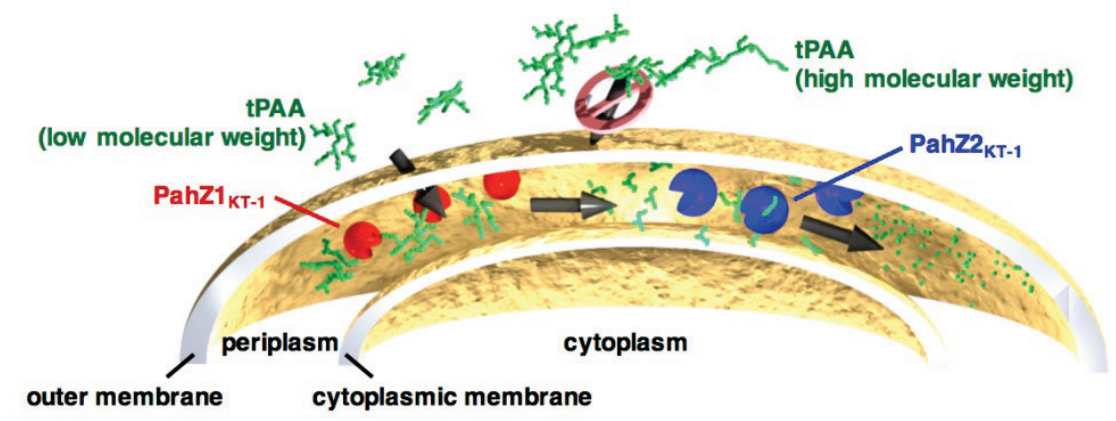

Figure 8. Possible mechanism for microbial degradation of tPAA by Sphingomonas sp. KT-1

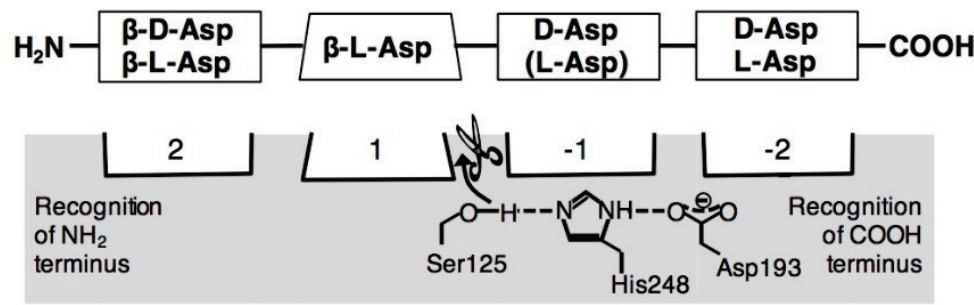

Figure 9. Schematic model of substrate recognition site of PahZ1 $1_{\mathrm{KP}-2}$

\subsubsection{Enzymes functionally and structurally related to PahZ1 ${ }_{\mathrm{KT}-1}$ and PahZ1 ${ }_{\mathrm{KP}-2}$}

Because PahZ1 enzymes specifically cleave novel $\beta$-linkages in tPAA, we focused on the functionally and structurally related enzymes that also recognize $\beta$-linkages in the polymers. Figure 10 shows the multi-alignment of the deduced amino acids of matured PahZ1 $1_{\mathrm{KT}-1}$ and PahZ1 $1_{\mathrm{KP}-2}$ and those of PHB depolymerases using ClustalW2 (GENETYX software). BLAST analysis revealed that the deduced amino acid sequence of matured PahZ1 $1_{\mathrm{KP}-2}$ was similar to that of PahZ1 $1_{\text {KT-1 }}$ (39\% identity in 264 aa) [129]. PahZ1 enzymes are classified into the $\alpha / \beta$-hydrolase_5 family, which includes putative PHB depolymerase (LpqC) from Bordetella parapertussis, based on the ESTHER database (http://bioweb.ensam.inra.fr/esther). As shown in Figure 10, the residues composing their catalytic triads, in which the Ser residue formed together with Asp and His residues, are conserved in PahZ1 $1_{\mathrm{KT}-1}, \mathrm{PahZ1}_{\mathrm{KP}-2}, \mathrm{PhaZ}_{\mathrm{AfaAE} 122}$, and 
PhaZ2 2 Ple. PHB depolymerases are monomer enzymes having an $\alpha / \beta$-hydrolase fold and cleave $\beta$-ester bonds in PHB via an endo-type process $[87,97,108]$. Early hydrolysis studies of oligo(3HB)s with well-defined sequences demonstrated the four subsites model at the substrate-binding site of PHB depolymerases, resembling that of PahZ1 $1_{\mathrm{KP}-2}[106,133,134]$.

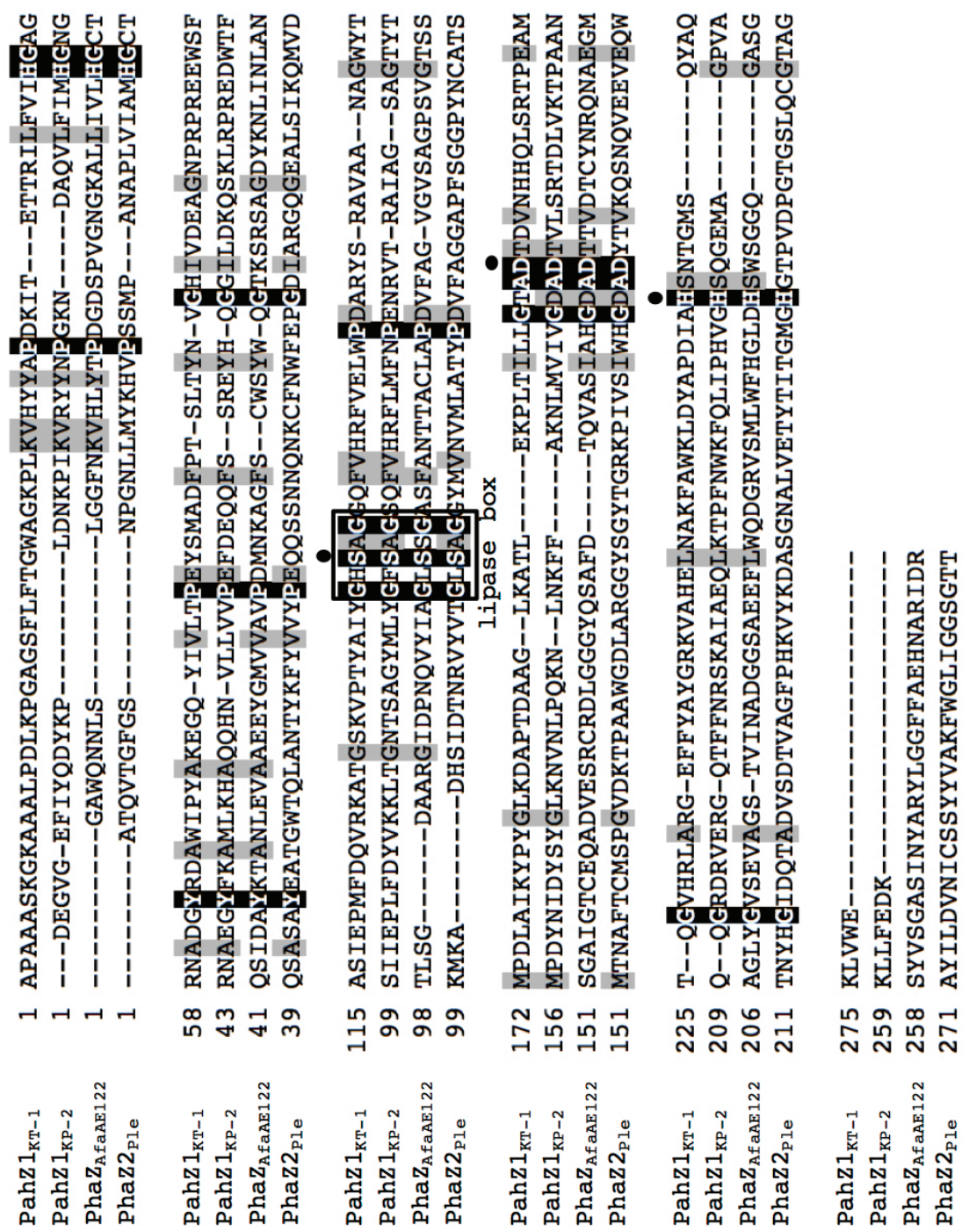

Figure 10. Multi-alignment of putative amino acids of matured PAA hydrolases-1 with those of matured PHB depolymerases using ClustalW2 (GENETYX software).

Sequences of PAA hydrolases-1 from Sphingomonas sp. KT-1 (PahZ1 $1_{\mathrm{KT}-1}$ ) and

Pedobacter sp. KP-2 (PahZ1 $1_{\mathrm{KP}-2}$ ) and PHB depolymerases from Alcaligenes faecalis AE122 (PhaZ $\mathrm{AfaAE} 122_{2}$ ) and Pseudomonas lemoignei (PhaZ2 $2_{\mathrm{Ple}}$ ) are shown. Identical and conserved amino acids are marked in black and gray, respectively. Box indicates lipase box. Proposed active site residues are marked by closed circles. 
In addition to the PAA-hydrolysing enzymes (PahZ1 ${ }_{\mathrm{KT}-1}, \mathrm{PahZ2}_{\mathrm{KT}-1}$, and PahZ1 $1_{\mathrm{KP}-2}$ ), five $\beta$-aminopeptidases (three BapA enzymes, one BapF enzyme, and one DmpA enzyme) hydrolyse short $\beta$-peptides and $\beta$-amino-acid containing peptides [135-142]. Their properties are listed in Table 2. The $\beta$-aminopeptidases are exo-type hydrolases that remove the $\beta$-amino acid unit from the $\mathrm{N}$-terminus of oligopeptides, amides, and esters. Based on the MEROPS database [143], they are classified into peptidase family P1, which includes aminopeptidases and self-processing proteins. The phylogenetic tree based on amino acid sequences showing relationships among PAA hydrolases-1, PHB depolymerases, and $\beta$-aminopeptidases strongly suggests that PahZ1 1 Кт-1 and PahZ1 $1_{\mathrm{KP}-2}$ are related to PhaZ $\mathrm{AfAAE}_{\mathrm{A} 22}$ and PhaZ2 $2_{\mathrm{Ple}}$ but not to $\beta$-aminopeptidases (Figure 11).

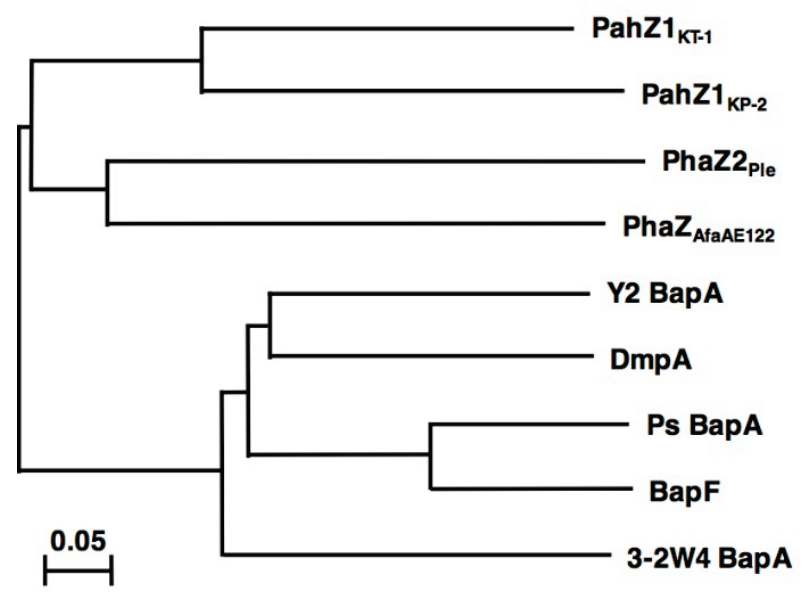

Figure 11. Neighbor-joining tree showing phylogenetic relationships among PAA hydrolases-1, PHB depolymerases, and $\beta$-aminopeptidases [Y2 BapA, Ps BapA

(BapA from Pseudomonas sp. MCI3434), 3-2W4 BapA, BapF (BapF from Pseudomonas aeruginosa PA01), and DmpA]. The scale bar represents the expected number of substitutions per amino acid position.

The chemical compounds containing $\beta$-amino-acid-derived substructures are part of a wide variety of bioactive secondary metabolites, such as coenzyme A, L-carnosine, taxol, microcystin-LR, and bestatin [142]. Moreover, $\beta$-L- and $\beta$-D-Asp units are found in various proteins, including $\alpha \mathrm{A}$ - and $\alpha \mathrm{B}$-crystallins, $\beta$-amyloid protein, and elastin from diverse tissues of elderly individuals [144]. There are no known natural peptides that are solely composed of $\beta$-amino acid units. Accordingly, compounds containing $\beta$-amino-acid-derived substructures and mixed $\alpha, \beta$-peptides produced in nature may serve as physiological substrates for PahZ1 and PahZ2 enzymes as well as DmpA and BapA enzymes. 


\subsubsection{Application of PAA hydrolase from Pedobacter sp. KP-2}

Enzymes have many benefits including high catalytic rates, lack of undesirable by-products, high enantio- and regioselectivities, and the ability to function under mild conditions, as catalysts of polymer synthesis compared with chemical catalysts. As commonly used proteases cleave and form $\alpha$-amide linkages, the resultant poly(amino acid)s synthesized by the proteases are composed of $\alpha$-units [145-151]. Due to the novel substrate specificity of PahZ1 enzymes, their application to enzyme-catalysed polymerization may result in the synthesis of $\beta$-linked PAA ( $\beta$-PAA) possessing the unexpected properties mentioned above. Thus, we applied PahZ1 $1_{\mathrm{KP}-2}$ to the enzyme-catalysed synthesis of $\beta$-PAA [152]. Matrix-assisted laser desorption/ionization time of flight mass spectrometry (MALDI-TOF MS) analysis demonstrated that the synthesized polymers ranged from $\mathrm{m} / \mathrm{z}=750$ to 2,500 and were composed of ethyl aspartate units containing either an ethyl ester or a free carboxyl end group at the carboxyl terminus. ${ }^{1} \mathrm{H}$ NMR analysis indicated that the synthesized polymer consisted of only $\beta$-amide linkages. Therefore, PahZ1 $1_{\mathrm{KP}-2}$ is a useful biocatalyst for $\beta$-peptide synthesis.

\subsection{CONCLUSION}

This chapter describes biopolymers containing a novel $\beta$-linkage in the main chain of their molecules, such as PHAs and PAA, and the enzymes involved in their metabolism. We focused on the development of PHA synthases and PHB depolymerases with higher activity or modified substrate-recognizing ability, as well as their applications. Furthermore, we described the structure-function relationships of PAA hydrolases and their applications.

Biodegradable and biobased biopolymers, such as PHAs and PAA, are desirable alternatives to petrochemical-derived polymers. For practical applications, three main factors must be considered, that is, reduction of their production cost, improvement of their performance, and their efficient recycling. Therefore, process improvements for biopolymer production, as well as biopolymer degradation, in particular, improvement of the enzymes used in the processes, are becoming increasingly important and widespread. Against this background, directed evolution of the enzymes involved in the biosynthesis and biodegradation of biopolymers will become the driving force to establish bioprocesses for the controlled production of biopolymers with the desired monomer compositions. In addition, systems-level analysis of metabolic, signalling, and regulatory networks in biopolymer-producing and biopolymer-degrading strains will provide new targets and strategies for process improvement, including tailor-made biopolymer production with desired monomer composition and molecular mass. 
Furthermore, the widespread use of biopolymers may require further development of their high value-added products and applications. In this chapter, we introduced several applications, including enzyme-catalysed polymerization and micropatterning and nanopatterning. In regards to enzyme-catalysed polymerization, custom-made enzymes generated by evolutionary engineering may affect the creation of novel high-performance biopolymers, such as $\beta$-peptides, in improved in vitro systems. The development of such enzymes is expected to catalyse dramatic breakthroughs in the industrial, pharmaceutical, and agricultural fields. Regarding the latter application, molecular patterning technologies have developed along two separate paths: serial writing [e.g., dip-pen nanolithography (DPN), 153] and parallel printing [e.g., $\mu \mathrm{Cp}, 154]$ and have enabled many applications, such as DNA microarrays and protein chips [155]. DNA microarrays have been used in expression profiling, mutation detection, diagnosis, and many other applications [156]. Protein chips haves also attracted interest in diagnostics, biosensor applications and library screening $[157,158]$. Recently, the two branches of patterning merged with the invention of massively parallel DPN and polymer-pen lithography [155,159]. In the field of nanotechnology, biocompatibility and low environmental impact of the byproducts are desired because of the increasing awareness of global environmental issues. Thus, fusion of the recently advanced patterning methods and the use of biopolymers and their improved enzymes will provide environmentally and people-friendly, high-throughput processing methods for soft interfaces with nanometre- to micrometre-sized architecture in the future.

\section{REFERENCES}

1. Y. Tokiwa, B.P. Calabia, C.U. Ugwu, S. Aiba, Int. J. Mol. Sci. 10 (2009) 37223742.

2. G.Q. Chen, M.K. Patel, Chem. Rev. 112 (2012) 2082-2099.

3. T. Candela, A. Fouet, Mol. Microbiol. 60 (2006) 1091-1098.

4. $\quad$ S.M. Thombre, B.D. Sarwade, J. Macromol. Sci., Part A: Pure Appl. Chem. $\mathbf{4 2}$ (2005) 1299-1315.

5. M. Lemoigne, Bull. Soc. Chim. Biol. 8 (1926) 770-782.

6. D. Seebach, M.G. Fritz, Int. J. Biol. Macromol. 25 (1999) 217-236.

7. R.N. Reusch, Int. J. Mol. Sci. 14 (2013) 10727-10748.

8. D. Jendrossek, D. Pfeiffer, Environ. Microbiol. 16 (2014) 2357-2373.

9. M. Pötter, A. Steinbüchel, Biomacromolecules 2 (2005) 552-560.

10. B.H. Rehm, Biothechnol. Lett. 28 (2006) 207-213.

11. D. Jendrossek, J. Bacteriol. 191 (2009) 3195-3202.

12. D. Jendrossek, R. Handrick, Annu. Rev. Microbiol. 56 (2002) 403-432.

13. D.Y. Kim, Y.H. Rhee, Appl. Microbiol. Biotechnol. 61 (2003) 300-308.

14. A. Steinbüchel, H.E. Valenthin, FEMS Microbiol. Lett. 128 (1995) 219-228.

15. Y. Doi, A. Steinbüchel (Eds.), Biopolymers vol. 4; Polyesters III: Applications and Commercial Products, WILEY-VCH Verlag GmbH \& Co. KGaA, Weinheim, Germany, 2002, P. 410. 
16. R.A.J. Verlinden, D.J. Hill, M.A. Kenward, C.D. Williams, I. Radecka, J. Appl. Microbiol. 102 (2007) 1437-1449.

17. G.Q. Chen, Chem. Soc. Rev. 38 (2009) 2434-2446.

18. R.N. Reusch, H.L. Sadoff, J. Bacteriol. 156 (1983) 778-788.

19. R.N. Reusch, T.W. Hiske, H.L. Sadoff, J. Bacteriol. 168 (1986) 553-562.

20. R.N. Reusch, Proc. Soc. Exp. Biol. Med. 191 (1989) 377-381.

21. D. Seebach, A. Brunner, H.M. Bürger, J. Schneider, R.N. Reusch, Eur. J. Biochem. 224 (1994) 317-328.

22. $\quad$ R.N Reusch, H.L. Sadoff, Proc. Natl. Acad. Sci. USA 85 (1988) 4176-4180.

23. R.N. Reusch, R. Huang, L.L. Bramble, Biophys. J. 69 (1995) 754-766.

24. S. Das, U.D. Lengweiler, D. Seebach, R.N. Reusch, Proc. Natl. Acad. Sci. USA 94 (1997) 9075-9079.

25. C.E. Castuma, R. Huang, A. Kornberg, R.N. Reusch, J. Biol. Chem. 270 (1995) 12980-12983.

26. R. Huang, R.N. Reusch, J. Bacteriol. 177 (1995) 486-490.

27. R.N. Reusch, Biochem. Moscow. 65 (2000) 280-295.

28. R. Huang, R.N. Reusch, J. Biol. Chem. 271 (1996) 22196-22202.

29. R.N. Reusch, Chem. Biodivers. 9 (2012) 2343-2366.

30. D. Dai, R.N. Reusch, Biochem. Biophys. Res. Commun. 374 (2008) 485-489.

31. R. Auras, B. Harte, S. Selke, Macromol. Biosci. 4 (2004) 835-864.

32. Y. Tokiwa, B.P. Calabia, Appl. Microbiol. Biotechnol. 72 (2006) 244-251.

33. Y.M. Zhao, Z.Y. Wang, J. Wang, H.Z. Mai, B. Yan, F. Yang, J. Appl. Polym. Sci. 91 (2004) 2143-2150.

34. A.C. Albertsson, U. Edlund, K. Stridsberg, Macromol. Symp. 157 (2000) 39-46.

35. H.R. Kricheldorf, Chemosphere 43 (2001) 49-54.

36. S. Taguchi, M. Yamada, K. Matsumoto, K. Tajima, Y. Sato, M. Munekata, K. Ohono, K. Kohda, T. Shimamura, H. Kambe, S. Obata, Proc. Natl. Acad. Sci. USA 105 (2008) 17323-17327.

37. K. Tajima, Y. Satoh, T. Satoh, R. Itoh, X. Han, S. Taguchi, T. Kakuchi, M. Munekata, Macromolecules 42 (2009) 1985-1989.

38. D. Seebach, A.K. Beck, D.J. Bierbaum, Chem. Biodivers. 1 (2004) 1111-1239.

39. D. Seebach, J. Gardiner, Acc. Chem. Res. 41 (2008) 1366-1375.

40. M.B. Freeman, Y.H. Paik, G. Swift, R. Wilczynski, S.K. Wolk, K.M. Yocom, in Hydrogels and biodegradable polymers for bioapplications, ACS Symposium Series, R.M. Ottenbrite, S.J. Huang, K. Park (Eds.), American Chemical Society, Washington DC, USA, 1996, p. 118-136.

41. C.L. Kim, A.P. Wheeler, L.P. Koskan, in Hydrophilic polymers, Advances in Chemistry 248, J.D. Glass (Ed.), American Chemical Society: Washington DC, USA, 1996, p. 99-111.

42. Y. Tang, A.P. Wheeler, in Biopolymers from polysaccharides and agroproteins, R. Gross, C. Scholz (Eds.), ACS Symposium Series, American Chemical Society, Washington DC, USA, 2001, p. 157-171.

43. W. Joentgen, N. Müller, A. Mitschker, H. Schmidt, in Polyamides and complex proteinaceous materials I, Biopolymers 7, S.R. Fahnestock, A. Steinbüchel (Eds.), WILEY-VCH Verlag GmbH \& Co. KGaA, Weinheim, Germany, 2003, p. 175-199.

44. S.M. Thombre, B.D. Sarwade, J. Macromol. Sci., Part A: Pure Appl. Chem. 42 (2005) 1299-1315.

45. H. Pivcova, V. Saudek, J. Drobnik, J. Vlasak, Biopolymers 20 (1981) 1605-1614.

46. H. Pivcova, V. Saudek, J. Drobnik, Polymer 23 (1982) 1237-1241. 
47. S.K. Wolk, G. Swift, Y.H. Paik, K.M. Yocom, R.L. Smith, E.S. Simon, Macromolecules 27 (1994) 7613-7620.

48. K. Matsubara, T. Nakato, M. Tomida, Macromolecules 31 (1998) 1466-1472.

49. T. Nakato, M. Yoshitake, K. Matsubara, M. Tomida, T. Kakuchi, Macromolecules 31 (1998) 2107-2113.

50. I.S. Aldor, J.D. Keasling, Curr. Opin. Biotechnol. 14 (2003) 475-483.

51. L.L. Madison, G.W. Huisman, Microbiol. Mol. Biol. Rev. 63 (1999) 21-53.

52. B.H.A. Rehm, Biochem. J. 376 (2003) 15-33.

53. S. Taguchi, Y. Doi, Macromol. Biosci. 4 (2004) 146-156.

54. C.T. Nomura, S. Taguchi, Appl. Microbiol. Biotechnol. 73 (2007) 969-979.

55. S. Taguchi, A. Maehara, K. Takase, M. Nakahara, H. Nakamura, Y. Doi, FEMS Microbiol. Lett. 198 (2001) 65-71.

56. S. Taguchi, H. Nakamura, T. Hiraishi, I. Yamato, Y. Doi, J. Biochem. 131 (2002) 801-806.

57. Y.M. Normi, T. Hiraishi, S. Taguchi, H. Abe, K. Sudesh, N. Najimudin, Y. Doi, Macromol. Biosci. 5 (2005) 197-206.

58. Y.M. Normi, T. Hiraishi, S. Taguchi, K. Sudesh, N. Najimudin, Y. Doi, Biotechnol. Lett. 27 (2005) 705-712.

59. T. Kichise, S. Taguchi, Y. Doi, Appl. Environ. Microbiol. 68 (2002) 2411-2419.

60. T. Tsuge, S. Watanabe, S. Saito, T. Hiraishi, H. Abe, Y. Doi, S. Taguchi, Macromol. Biosci. 7 (2007) 846-854.

61. A.A. Amara, A. Steinbüchel, B.H.A. Rehm, Appl. Microbiol. Biotechnol. 59 (2002) 477-482.

62. K. Matsumoto, K. Takase, Y. Yamamoto, Y. Doi, S. Taguchi, Biomacromolecules 10 (2009) 682-685.

63. K. Takase, S. Taguchi, Y. Doi, J. Biochem. 133 (2003) 139-145.

64. K. Takase, K. Matsumoto, S. Taguchi, Y. Doi, Biomacromolecules 5 (2004) 480485.

65. T. Tsuge, Y. Saito, M. Narike, K. Muneta, Y.M. Normi, Y. Kikkawa, T. Hiraishi, Y. Doi, Macromol Biosci. 4 (2004) 963-970.

66. K. Matsumoto, K. Takase, E. Aoki, Y. Doi, S. Taguchi, Biomacromolecules 6 (2005) 99-104.

67. K. Matsumoto, E. Aoki, K. Takase, Y. Doi, S. Taguchi, Biomacromolecules 7 (2006) 2436-2442.

68. F. Shozui, K. Matsumoto, T. Sasaki, S. Taguchi, Appl. Microbiol. Biotechnol. 84 (2009) 1117-1124.

69. K. Matsumoto, R. Nagao, T. Murata, Y. Arai, T. Kichise, H. Nakashita, S. Taguchi, H. Shimada, Y. Doi, Biomacromolecules 6 (2005) 2126-2130.

70. K. Matsumoto, T. Murata, R. Nagao, C.T. Nomura, S. Arai, K. Takase, H. Nakashita, S. Taguchi, H. Shimada, Biomacromolecules 10 (2009) 686-690.

71. D.K. Solaiman, J. Ind. Microbiol. Biotechnol. 30 (2003) 322-326.

72. D.S. Sheu, C.Y. Lee, J. Bacteriol. 186 (2004) 4177-4184.

73. N. Niamsiri, S.C. Delamarre, Y.R. Kim, C.A. Batt, Appl. Environ. Microbiol. 70 (2004) 6789-6799.

74. H.E. Valentin, A. Steinbüchel, Appl. Microbiol. Biotechnol. 40 (1994) 699-709.

75. W. Yuan, Y. Jia, J.M. Tian, K.D. Snell, U. Muh, A.J. Sinskey, R.H. Lambalot, C.T. Walsh, J. Stubbe, Arch. Biochem. Biophys. 394 (2001) 87-98.

76. M. Yamada, K. Matsumoto, K. Shimizu, S. Uramoto, T. Nakai, F. Shozui, S. Taguchi, Biomacromolecules 11 (2010) 815-819. 
77. X. Han, Y. Satoh, T. Satoh, K. Matsumoto, T. Kakuchi, S. Taguchi, T. Dairi, M. Munekata, K. Tajima, Appl. Microbiol. Biotechnol. 92 (2011) 509-517.

78. K. Matsumoto, S. Terai, A. Ishiyama, J. Sun, T. Kabe, Y. Song, J.M. Nduko, T. Iwata, S. Taguchi, Biomacromolecules 14 (2013) 1913-1918.

79. K. Matsumoto, A. Ishiyama, T. Shiba, S. Taguchi, J. Biotechnol. 156 (2011) 214217.

80. K. Matsumoto, T. Shiba, Y. Hiraide, S. Taguchi, ACS Biomater. Sci. Eng. (2016) Articles ASAP (DOI: 10.1021/acsbiomaterials.6b00194).

81. T.H. Yang, Y.K. Jung, H.O. Kang, T.W. Kim, S.J. Park, S.Y. Lee, Appl. Microbiol. Biotechnol. 90 (2011) 603-614.

82. K. Tajima, X. Han, Y. Satoh, A. Ishii, Y. Araki, M. Munekata, S. Taguchi, Appl. Microbiol. Biotechnol. 94 (2012) 365-376.

83. A. Ochi, K. Matsumoto, T. Ooba, K. Sasaki, T. Tsuge, S. Taguchi, Appl. Microbiol. Biotechnol. 97 (2012) 3441-3447.

84. K. Matsumoto, S. Taguchi, Curr. Opin. Biotechnol. 24 (2013) 1054-1060.

85. K. Matsumoto, S. Taguchi, Appl. Microbiol. Biotechnol. 97 (2013) 8011-8021.

86. M. Knoll, T.M. Hamm, F. Wagner, V. Martines, J. Pleiss, BMC Bioinform. 10 (2009) 89-96.

87. D. Jendrossek, R. Handrick, Annu. Rev. Microbiol. 56 (2002) 403-432.

88. T. Hiraishi, S. Taguchi, Mini-Rev. Org. Chem. 6 (2009) 44-54.

89. T. Hiraishi, S. Taguchi, (2013) in Protein engineering - technology and application, T. Ogawa (Ed.), InTech, Rijeka, Croatia, 2013, p. 133-165.

90. J.D. Bloom, M.M Meyer, P. Meinhold, C.R. Otey, D. Macmillan, F.H. Arnold, Curr. Opin. Struc. Biol. 15 (2005) 447-452.

91. L.G. Otten, W.J. Quax, Biomol. Eng. 22 (2005) 1-9.

92. T.W. Johannes, H. Zhao, Curr. Opin. Microbiol. 9 (2006) 261-267.

93. H. Zhao, K. Chockalingam, Z. Chen, Curr. Opin. Biotechnol. 13 (2002) 104-110.

94. T. Hiraishi, Y. Hirahara, Y. Doi, M. Maeda, S. Taguchi, Appl. Environ. Microbiol. 72 (2006) 7331-7338.

95. T. Hiraishi, N. Komiya, N. Matsumoto, H. Abe, M. Fujita, M. Maeda, Biomacromolecules 11 (2010) 113-119.

96. S. Miyazaki, K. Takahashi, M. Shiraki, T. Saito, Y. Tezuka, K. Kasuya, J. Polym. Environ. 8 (2002) 175-182.

97. T. Hisano, K. Kasuya, Y. Tezuka, N. Ishii, T. Kobayashi, M. Shiraki, E. Oroudjev, H. Hansma, T. Iwata, Y. Doi, T. Saito, K. Miki, J. Mol. Biol. 356 (2006) 993-1004.

98. R. Handrick, S. Reinhardt, M.L. Focarete, M. Scandola, G. Adamus, M. Kowalczuk, D. Jendrossek, J. Biol. Chem. 276 (2001) 36215-36224.

99. T. Hiraishi, N. Komiya, M. Maeda, Polym. Degrad. Stab. 95 (2010) 1370-1374.

100. S.Y. Lee, J.H. Choi, Z. Xu, Trends Biotechnol. 21 (2003) 45-52.

101. M. Olsen, B. Iverson, G. Georgiou, Curr. Opin. Biotechnol. 11 (2000) 331-337.

102. L.-T. Tan, T. Hiraishi, K. Sudesh, M. Maeda, Appl. Microbiol. Biotechnol. 97 (2013) 4859-4871

103. T. Hiraishi, K. Yamashita, M. Sakono, J. Nakanishi, L.-T. Tan, K. Sudesh, H. Abe, M. Maeda, Macromol. Biosci. 12 (2012) 218-224.

104. H. Zhao, W. Zha, Nat. Protoc. 1 (2006) 1865-187.

105. L.-T. Tan, T. Hiraishi, K. Sudesh, M. Maeda, Appl. Microbiol. Biotechnol. 98 (2014) 7061-7068.

106. B.M. Bachmann, D. Seebach, Macromolecules 32 (1999) 1777-1784. 
107. A.C. Papageorgiou, S. Hermawan, C.B. Singh, D. Jendrossek, J. Mol. Biol. 382 (2008) 1184-1194.

108. S. Wakadkar, S. Hermawan, D. Jendrossek, A.C. Papageorgiou, Acta Cryst. F66 (2010) 648-654.

109. A.C. Albertsson, R.V. Srivastava, Adv. Drug Deliv. Rev. 60 (2008) 1077-1093.

110. A. Kumar, R.A. Gross, D. Jendrossek, J. Org. Chem. 65 (2000) 7800-7806.

111. Y. Suzuki, T. Ohura, K. Kasuya, K. Toshima, Y. Doi, S. Matsumura, Chem. Lett. (2000) 318-319.

112. Y. Suzuki, S. Taguchi, T. Saito, K. Toshima, S. Matsumura, Y. Doi, Biomacromolecules 2 (2001) 541-544.

113. Y. Suzuki, S. Taguchi, T. Hisano, K. Toshima, S. Matsumura, Y. Doi, Biomacromolecules 4 (2003) 537-543.

114. M. Santos, J. Gangoiti, M.J. Llama, J.L. Serra, H. Keul, M. Möller, J. Mol. Catal. B Enzym. 77 (2012) 81-86.

115. M. Santos, J. Gangoiti, M.J. Llama, J.L. Serra, H. Keul, M. Möller, Appl. Microbiol. Biotechnol. 97 (2013) 211-222.

116. Z. Nie, E. Kumacheva, Nat. Mater. 7 (2008) 277-290.

117. Z. Mao, M. Ganesh, M. Bucaro, I. Smolianski, R.A. Gross, A.M. Lyons, Biomacromolecules 15 (2014) 4627-4636.

118. I. Khana, J.R. Duttaa, R. Ganesan, J. Cellular Biotechnol. 1 (2015/2016) 131144.

119. Y. Kikkawa, M. Fukuda, N. Ichikawa, A. Kashiwada, K. Matsuda, M. Kanesato, T. Hiraishi, J. Mater. Chem. A 1 (2013) 4667-4670.

120. J.P. Park, K.-B. Lee, S.J. Lee, T.J. Park, M.G. Kim, B.H. Chung, Z.-W. Lee, I.S. Choi, S.Y. Lee, Biotechnol. Bioeng. 92 (2005) 160-165.

121. T.J. Park, J.P. Park, S.J. Lee, H.J. Hong, S.Y. Lee, Biotechnol. Bioproc. Eng. 11 (2006) 173-177.

122. T.J. Park, S.M. Yoo, K.C. Keum, S.Y. Lee, Anal. Bioanal. Chem. 393 (2009) 16391647.

123. T. Hiraishi, M. Maeda, Appl. Microbiol. Biotechnol. 91 (2011) 895-903.

124. T. Hiraishi, Appl. Microbiol. Biotechnol. 100 (2016) 1623-1630.

125. K. Tabata, K. Kasuya, H. Abe, K. Masuda, Y. Doi, Appl. Environ. Microbiol. 65 (1999) 4268-4270.

126. K. Tabata, H. Abe, Y. Doi, Biomacromolecules 1 (2000) 157-161.

127. K. Tabata, M. Kajiyama, T. Hiraishi, H. Abe, I. Yamato, Y. Doi, Biomacromolecules 2 (2001) 1155-1160.

128. T. Hiraishi, M. Kajiyama, K. Tabata, H. Abe, I. Yamato, Y. Doi, Biomacromolecules 4 (2003) 1285-1292.

129. T. Hiraishi, E. Masuda, N. Kanayama, M. Nagata, Y. Doi, H. Abe, M. Maeda, Macromol. Biosci. 9 (2009) 10-19.

130. T. Hiraishi, M. Kajiyama, K. Tabata, I. Yamato, Y. Doi, Biomacromolecules 4 (2003) 80-86.

131. T. Hiraishi, M. Kajiyama, I. Yamato, Y. Doi, Macromol. Biosci. 4 (2004) 330-339.

132. T. Hiraishi, H. Abe, M. Maeda, AMB Express 5 (2015) 31.

133. T. Hiraishi, T. Ohura, S. Ito, K. Kasuya, Y. Doi, Biomacromolecules 1 (2000) 320324.

134. T.M. Scherer, R.C. Fuller, S. Goodwin, R.W. Lenz, Biomacromolecules, 1 (2000) 577-583. 
135. B. Geueke, K. Namoto, D. Seebach, H.P.E. Kohler, J. Bacteriol. 187 (2005) 59105917.

136. H. Komeda, Y. Asano, FEBS J. 272 (2005) 3075-3084.

137. B. Geueke, T. Heck, M. Limbach, D. Seebach, H.P.E. Kohler, FEBS J. 273 (2006) 5261-5272.

138. T. Heck, M. Limbach, B. Geueke, M. Zacharas, J. Gardiner, H.P.E. Kohler, D. Seebach, Chem. Biodivers. 3 (2006) 1325-1348.

139. B. Geueke, H.P.E. Kohler, Appl. Microbiol. Biotechnol. 74 (2007) 1197-1204.

140. T. Heck, H.P.E. Kohler, M. Limbach, O. Flcgel, D. Seebach, B. Geueke, Chem. Biodivers. 4 (2007) 2016-2030.

141. V. Fuchs, K.E. Jaeger, S. Wilhelm, F. Rosenau, World J. Microbiol. Biotechnol. 27 (2011) 713-718.

142. T. Heck, B. Geueke, H.P.E. Kohler, Chem. Biodivers. 9 (2012) 2388-2409.

143. N.D. Rawlings, A.J. Barrett, A. Bateman, Nucleic Acids Res. 38 (2010) D227D233.

144. $\quad$ N. Fujii, Y. Kaji, N. Fujii, J. Chromatogr. B 879 (2011) 3141-3147.

145. K. Aso, T. Uemura, Y. Shiokawa, Agric. Biol. Chem. 52 (1988) 2443-2449.

146. T. Uemura, M. Fujimori, H.H. Lee, S. Ikeda, K. Aso, Agric. Biol. Chem. 54 (1990) 2277-2281.

147. S. Matsumura, Y. Tsushima, N. Otozawa, S. Murakami, K. Toshima, G. Swift, Macromol. Rapid Commun. 20 (1999) 7-11.

148. H. Uyama, T. Fukuoka, I. Komatsu, T. Watanabe, S. Kobayashi, Biomacromolecules 3 (2002) 318-323.

149. Y. Soeda, K. Toshima, S. Matsumura, Biomacromolecules 4 (2003) 196-203.

150. G. Li, A. Vaidya, K. Viswanathan, J. Cui, W. Xie, W. Gao, R.A. Gross, Macromolecules 39 (2006) 7915-7921

151. G. Li, V.K. Raman, W. Xie, R.A. Gross, Macromolecules 41 (2008) 7003-7012.

152. T. Hiraishi, E. Masuda, D. Miyamoto, N. Kanayama, H. Abe, M. Maeda, Macromol. Biosci. 11 (2011) 187-191.

153. S.H. Hong, J. Zhu, C.A. Mirkin, Science 286 (1999) 523-525.

154. A. Kumar, G.M. Whitesides, Appl. Phys. Lett. 63 (1993) 2002-2004.

155. A.B. Braunschweig, F. Huo, C.A. Mirkin, Nat. Chem. 1 (2009) 353-358.

156. S.M. Yoo, D.C. Keum, S.Y. Yoo, J.Y. Choi, K.H. Chang, N.C. Yoo, W.M. Yoo, J.M. Kim, D. Lee, S.Y. Lee, Biotechnol. Bioproc. Eng. 9 (2004) 93-99.

157. D.S. Wilson, S. Nock, Curr. Opin. Chem. Biol. 6 (2002) 81-85.

158. T.J. Park, M.Y. Seung, K.C. Keum, S.Y. Lee, Anal. Bioanal. Chem. 393 (2009) 1639-1647.

159. C.D. O'Connell, M.J. Higgins, S.E. Moulton, G.G. Wallace, J. Mater. Chem. C 3 (2015) 6431-6444. 